(C) 2000 International Press

Adv. Theor. Math. Phys. 4 (2000) 679-745

\title{
Curvature Singularities: the Good, the Bad, and the Naked
}

\author{
Steven S. Gubser \\ Joseph Henry Laboratories, Princeton University \\ Princeton, NJ 08544
}

\begin{abstract}
Necessary conditions are proposed for the admissibility of singular classical solutions with 3+1-dimensional Poincaré invariance to five-dimensional gravity coupled to scalars. Finite temperature considerations and examples from AdS/CFT support the conjecture that the scalar potential must remain bounded above for a solution to be physical. Having imposed some restrictions on naked singularities allows us to comment on a recent proposal for solving the cosmological constant problem.
\end{abstract}

e-print archive: http://xxx.lanl.gov/hep-th/0002160 


\section{Introduction}

In AdS/CFT [1, 2, 3] (see [4] for a review), bulk geometries which are only asymptotically $A d S_{5}$ near the timelike boundary are dual to relevant deformations of the CFT or to non-conformal vacua. Far from the timelike boundary, various singularities might arise. There must be some restrictions on the type of singularity which is allowed: for instance, we take it as obvious that negative mass Schwarzschild should be excluded on the grounds of vacuum stability [5]. The goal of this paper is to address the question of what singularities are allowed in geometries of the form

$$
\begin{aligned}
d s^{2} & =e^{2 A(r)}\left(-d t^{2}+d \vec{x}^{2}\right)+d r^{2} \\
\vec{\varphi} & =\vec{\varphi}(r),
\end{aligned}
$$

where by assumption $A(r) \sim r / L$ and $\vec{\varphi}(r) \sim$ (const) for large $r . L$ is the radius of curvature of the asymptotically $A d S_{5}$ region. Geometries of the form (1) arise in the gauged supergravity description of certain vacuum states on the Coulomb branch of $\mathcal{N}=4$ super-Yang-Mills theory $[6,7,8,9,10]$, and are also suspected to be the supergravity duals of most renormalization group flows arising from relevant deformations of the lagrangian of the CFT $[11,12,13,14,15,16,17,18,19,20,21$, $22,23]$.

The classical equations of motion, plus either positivity of the scalar kinetic terms or the more general "null energy" condition, together imply $A^{\prime \prime}(r) \leq 0[14,18]$. Since we assume $A(r) \sim r / L$ with $L>0$ for large $r, A(r)$ must in fact be monotonically increasing with $r$. Thus a curvature singularity can arise for the metric (1) if $A(r) \rightarrow-\infty$ at some finite $r_{0}{ }^{1}$

\footnotetext{
${ }^{1}$ Singularities where $A(r) \rightarrow-\infty$ at finite $r_{0}$ are the only type that will be encountered in this paper, but for the sake of completeness let us review three other possibilities. First, it could be that $A(r)$ is defined for all $r$, but $A^{\prime}(r) \rightarrow \infty$ as $r \rightarrow-\infty$. Supersymmetric examples with this behavior arise in certain $U(1)$ gauged supergravities. Second, an $S^{5}$ shell of D3-branes produces a near-horizon geometry which is $A d S_{5} \times S^{5}$ outside the shell and flat $\mathbf{R}^{10}$ inside the shell $[24,25]$. The shell itself produces a curvature singularity at finite $A(r)$. Similar singularities arise in Horava-Witten theory compactified on a Calabi-Yau three-fold with M5-branes in the bulk (see for example [26]): in the five-dimensional description, there is a jump in $A^{\prime}(r)$ across each M5-brane. This sort of singularity also arises in type $\mathrm{I}^{\prime}$ with D8-branes in the bulk. Finally, there can be a codimension one orientifold plane at
} 
The UV-IR relation $[27,28]$ suggests that curvature singularities that arise as $A(r) \rightarrow-\infty$ represent non-trivial infrared physics in the dual field theory. (Recall that $A(r)=\frac{1}{2} \log \left|g_{t t}\right|$ is roughly the log of the energy scale). This interpretation is natural from the point of view of the holographic renormalization group [22]. Suppose the field theory vacuum is one which can support finite temperature: by this we mean that the vacuum can be approached smoothly in a $T \rightarrow 0$ limit, with no zero temperature phase transition. Then a putative dual description in supergravity should also be able to support finite temperature, in the form of a black hole horizon which "cloaks" the curvature singularity. As the temperature is lowered to zero, the horizon should retreat until the original singular geometry is recovered. Thus we arrive at a weak form of Cosmic Censorship: the only curvature singularities allowed in geometries of the form (1) are those which can be obtained as limits of regular black holes.

Even this statement is too strong: there are interesting vacua in field theory which cannot support finite temperature. Examples include states on the Coulomb branch of $\mathcal{N}=4$ gauge theory. The dual geometries are typically singular in five dimensions. Any finite temperature, no matter how small, would draw a Coulomb branch state to the origin of moduli space, where conformal invariance is recovered in the zero temperature limit. Correspondingly, finite temperature in the bulk should pull the dual singular geometries back to AdS-Schwarzschild.

As detectives in search of a useful rule for distinguishing good singularities from bad, let us then ask the following two questions. (1) Do the singular five-dimensional geometries corresponding to Coulomb branch states share any common features with geometries which are limits of regular black holes? (2) Can we find other clear examples which could help us establish the identifying marks of healthy singularities as opposed to pathological ones? The main goal of this paper is to answer both questions affirmatively, and in fact to argue that

Large curvatures in geometries of the form (1) are allowed only if the scalar potential is bounded above in the solution.

This conjecture is peculiar in that it is the opposite of the naive intuition finite $A(r)$ on which the five-dimensional spacetime ends. Again the examples are type $\mathrm{I}^{\prime}$ and Horava-Witten theory. 
from classical relativity. The dominant energy condition, for example, constrains the scalar potential to be non-negative-not just in solutions, but in the lagrangian. (This of course rules out an $A d S_{5}$ solution, since the scalar potential is the cosmological constant). Nevertheless, it will be proven that (2) is indeed a necessary condition for a bulk geometry to support finite temperature in the form of a black hole horizon. It will also be shown that (2) correctly predicts some non-trivial aspects of the vacuum structure of mass-deformed $\mathcal{N}=4$ gauge theory.

It is important to bear in mind that (2) is a constraint on solutions, not on the five-dimensional lagrangian. In point of fact, the scalar potential for $d=5 \mathcal{N}=8$ gauged supergravity (from which all our examples will be drawn) is unbounded both above and below. There are solutions where scalars diverge near the singularity in such a way that the scalar potential goes to $+\infty$. These are bad, according to (2). There are others where the scalar potential goes to $-\infty$. These are good. Thanks to AdS/CFT, we can not only demonstrate that the good singularities have sensible field theory duals, but in most cases even trace the sickness of the bad singularities back to some definite pathology in the dual field theory.

A complementary line of attack, which will not be pursued in this work but is potentially very fruitful, is to resolve singularities in metrics of the form (1) by lifting them to ten dimensions and finding a configuration of branes that produces the ten-dimensional geometry (possibly in some near-horizon, large $N$ limit). This is in general a difficult task, but when it can be carried out, it provides a satisfying account of how string theory resolves a singularity. A goal of the present paper is to try to anticipate which five-dimensional singularities will admit a brane resolution. The methods should be equally applicable to Calabi-Yau compactifications of Horava-Witten theory where one endof-the-universe brane has retreated to infinite redshift. This is a case where our understanding of the microscopic eleven-dimensional theory is so incomplete that supergravity methods, combined with consistency conditions such as anomaly inflow, may be the only tools available.

One may legitimately inquire why any method based on five-dimensional supergravity should have predictive power regarding the nature of singular solutions. This is where AdS/CFT helps: confusing singularities on the AdS side are often transparent in the field theory dual. 
In the end, however, the hope is that (2) is a robust result that will survive beyond the approximations in which we have studied it, and even beyond AdS/CFT. The nature of a local singularity should be independent of the geometry far from it, so having an asymptotically $A d S_{5}$ region may be just a crutch for calculation. The physics of the naked singularity should be as independent of the asymptotically $A d S_{5}$ region as infrared effective theories are of their detailed microscopic origins.

The organization of the paper is as follows. Section 2 is a more technical introduction in which conventions are set and a method for generating solutions is reviewed. In section 3 we discuss the motivations for (2), and we explain some related conditions which are useful when looking at examples. We also discuss the infrared asymptotics of generic solutions and make some speculations regarding phase transitions. In section 4 we review three examples of asymptotically $A d S_{5}$ geometries which arise as deformations of $\mathcal{N}=4$ super-Yang-Mills theory by a relevant operator. In section 5 we consider linearized fluctuations around a general asymptotically $A d S_{5}$ background and propose a generalization of the Breitenlohner-Freedman bound. Concluding remarks and some further conjectures are presented in section 6 . In the appendix we remark on brane-world scenarios in which there is a curvature singularity parallel to our world, at some finite proper distance from it.

\section{Background}

All the geometries we will consider are classical solutions to the equations of motion following from an action

$$
S=\int d^{5} x \sqrt{g}\left[\frac{1}{4} R-\frac{1}{2}(\partial \vec{\varphi})^{2}-V(\vec{\varphi})\right] .
$$

Usually it will be assumed that the potential $V(\vec{\varphi})$ has a local maximum at $\vec{\varphi}=0$. Our main example of such an action is the gravity-plusscalars sector of $d=5 \mathcal{N}=8$ gauged supergravity [29, 30]. Here the 42 scalars $\vec{\varphi}$ parametrize the coset $E_{6(6)} / U S p(8)$, and $V(\vec{\varphi})$ is a $S O(6) \times$ $S L(2, \mathbf{R})$-invariant function on this coset. The kinetic term would be more accurately represented as $G_{I J}(\vec{\varphi}) \partial \varphi^{I} \partial \varphi^{J}$, where $G_{I J}(\vec{\varphi})$ is the natural sigma model metric for the coset. In the general discussion 
that follows, we will suppress factors of $G_{I J}$ and its inverse. Restoring them does not make any difference to the essential points of the story as long as $G_{I J}$ is a smooth function of $\vec{\varphi}$, which is true for $d=5$ $\mathcal{N}=8$ gauged supergravity. In the examples of section 4 we will work on submanifolds of $E_{6(6)}$ where the metric is flat, so one may find a basis of scalars where $G_{I J}=\delta_{I J}$. We will call such scalars canonically normalized.

The 40 scalars that participate in $V(\vec{\varphi})$ are tachyonic at the maximally supersymmetric point representing unperturbed $\mathcal{N}=4$ superYang-Mills theory. In AdS/CFT they correspond to dimension 2 operators in the $\mathbf{2 0}^{\prime}$ of $S O(6)$ and dimension 3 operators in the $\mathbf{1 0}+\overline{\mathbf{1 0}}$. The remaining scalars are the exactly massless dilaton and axion, corresponding to the exactly marginal complex operator $\operatorname{tr}\left(F^{2}+i F \tilde{F}+\ldots\right)$. These massless scalars will not participate in the geometries we consider. Our remarks can be applied more generally to less supersymmetric AdS/CFT duals, the key point being that the scalars in question are tachyonic at $\vec{\varphi}=0$.

It is often useful to take a potential of the form

$$
V(\vec{\varphi})=\frac{1}{8}\left(\frac{\partial W}{\partial \vec{\varphi}}\right)^{2}-\frac{1}{3} W(\vec{\varphi})^{2}
$$

where $W$ is some appropriate function of the scalars. In $U(1)$ gaugings of $d=5 \mathcal{N}=2$ supergravity, $W$ can be identified with the superpotential. In $[16,18,8,20]$ it was shown that the potential of $d=5 \mathcal{N}=8$ gauged supergravity also assumes the form (4) on restricted subspaces of the scalar manifold, with $W$ identified as an eigenvalue of an $S O(6) \times S L(2, \mathbf{R})$-invariant $U S p(8)$ matrix $W_{a b}$, defined on $E_{6(6)} / U S p(8)$, which serves as a generalized superpotential in the sense that the gravitino variation has the form $\delta \psi_{\mu a}=\mathcal{D}_{\mu} \epsilon_{a}-\frac{1}{6} g W_{a b} \gamma_{\mu} \epsilon^{b}$ (see $[29,18]$ for further details).

In $[31,32,33]$, the form (4) was found to be useful, independent of supersymmetry, for generating solutions of the form (1) and investigating their properties. Indeed, given $V(\vec{\varphi})$, the equation (4) can be regarded as a PDE for $W$, to be solved with arbitrary but specified initial conditions. It was observed in [22] that the $W$ so obtained is related to Hamilton's principle function. Of the possible forms for $W$ satisfying (4), only one (or at most discretely many) can be related 
to the supersymmetry transformations. However, if (4) can be solved systematically, then it is possible to generate any solution of the form (1) (supersymmetric or not) to the equations of motion by solving the first order equations

$$
\vec{\varphi}^{\prime}=\frac{1}{2} \frac{\partial W}{\partial \vec{\varphi}} \quad A^{\prime}=-\frac{1}{3} W(\vec{\varphi}) .
$$

Except in section 5, primes will indicate derivatives with respect to $r$. It can happen that $W$ is not a single-valued function of $\vec{\varphi}$, but rather a multi-sheeted graph in the allowed region of $W-\vec{\varphi}$ space where $V(\vec{\varphi})+\frac{1}{3} W(\vec{\varphi})^{2} \geq 0$. In such a case, (5) must be supplemented with selection rules for going from one branch to another when the boundary of the allowed region is reached (see [33] for a more detailed discussion). This subtlety will not be a concern in this paper.

The prima facia evidence that the solution space of (4) together with (5) coincides with the solution space of the equations of motion following from (3) is that the number of integration constants is the same. In view of the realization [22] that (5) can be recovered in HamiltonJacobi theory, there can be no question that (4) together with (5) are nothing more nor less than a fancy rewriting of the equations of motion. Nevertheless, it will be instructive to follow through the counting of integration constants. Suppose there are $n$ scalars. The equations of motion consist of $n$ second order differential equations, one for each scalar, plus one first order constraint from the $G^{r}{ }_{r}$ Einstein equation. That means $2 n+1$ integration constants. In the first order formalism, $n$ of these integrations constants are tucked into $W$ as constants of the motion; $n$ more come from the first order equations for the scalars; and the last comes from the equation for $A^{\prime}$ in (5), which is equivalent to the $G_{r}^{r}$ Einstein equation if the first order equations for the scalars hold.

There are various reasons to think that the space of solutions of the form (1) to the equations of motion following from (3) is too big-that not all solutions are physical. First, from a field theory perspective, it is possible that the vacuum is ill-defined. This would happen, for instance, if the effective potential in the field theory were unbounded below. It was suggested in [15] that exactly this problem was responsible for the unphysical nature of the $S O(5)$ critical point. To flow to the $S O(5)$ point, it is necessary to deform the $\mathcal{N}=4$ lagrangian by a 
term $m^{2} \operatorname{tr}\left(-X_{1}^{2}-X_{2}^{2}-X_{3}^{2}-X_{4}^{2}-X_{5}^{2}+5 X_{6}^{2}\right)$. This means the effective potential $V_{\text {eff }}$ in the field theory is unbounded below. This pathology manifests itself in five-dimensional gauged supergravity as a violation of the Breitenlohner-Freedman bound $[34,35]$ at the $S O(5)$ critical point. Another pathology which one could imagine in field theory is attempting to assign a negative vacuum expectation value (VEV) to a positive definite operator. In section 4 we will encounter examples where this problem arises.

Generic solutions of the form (1) to the equations of motion following from (3) have curvature singularities at finite $r$. A very few, such as $A d S_{5}$ itself and the RG flows of $[14,15,18]$, have curvatures everywhere bounded. We believe we are on safe ground when we assert that not all naked singularities are physical, even if we demand $3+1$ dimensional Poincaré invariance and/or some unbroken supersymmetry. Indeed, some of the Coulomb branch flows explored in $[8,36,10]$ have an unphysical property when they were lifted to ten dimensions: the brane distributions involve branes both of positive tension and positive charge, and of negative tension and negative charge. A similar problem was beautifully resolved in [37], but for Coulomb branch states it is obscure what enhancement of symmetry could play a relevant role. In section 4 we will encounter other pathologies in geometries which violate (2). In short, it seems hard to make a convincing case that all the nakedly singular, Poinaré-invariant geometries that we can generate as solutions to the equations of motion are physical.

We would however take the position that there are physically allowable naked singularities in AdS/CFT, representing interesting infrared physics in the dual field theory-only supergravity has limited calculation power in their vicinity. The repulson/enhançon of [37] is one example; the dual to $\mathcal{N}=1$ super-Yang-Mills theory [20] is another, which we will have more to say about later. In general, it is a nontrivial exercise to lift five-dimensional solutions to ten dimensions and find a brane configuration which realizes the geometry in some appropriate limit. And it is painful to proceed example-by-example, looking for known pathologies, like negative VEV's for positive definite operators, or imaginary ten-dimensional metrics. Thus it is useful to have a criterion such as (2), which is easy to apply and seems fairly reliable. 


\section{Motivating the criterion}

In this section we will provide two motivations for (2). The first is that it is a necessary condition for the existence of near-extremal generalizations of a solution to (5). We will prove this assertion in section 3.1. By near-extremal generalizations we mean a family of black hole solutions, with horizons hiding the naked singularity, which converge to the Poincare invariant solution in an appropriate topology. The Hawking temperature of the horizon is identified with finite temperature in the dual field theory.

Finite temperature in field theory serves as an infrared cutoff in the sense that it masks physics at scales lower than the temperature. A naked singularity that can be hidden behind a black hole horizon is a signal of non-trivial but sensible infrared physics in the dual field theory. A naked singularity that cannot be so hidden may indicate a pathology - the absence of a well-defined dual field theory, or a field theory in an unphysical vacuum state. If we approach a "good" naked singularity by taking a limit of regular black holes, the dual picture is that the infrared cutoff (finite temperature) is being removed. The calculational power of AdS/CFT may be limited in such a circumstance because of the large curvatures near the singularity.

The second motivation for (2) is an examination of states on the Coulomb branch of $\mathcal{N}=4$ super-Yang-Mills theory. Finite temperature draws Coulomb branch states to the origin of moduli space, where all operators have vanishing vacuum expectation values. Thus if one can demonstrate (2) for Coulomb branch states, it is a check of the conjecture which is orthogonal to finite temperature considerations. In fact there is a class of Coulomb branch vacua which can be described in $d=5 \mathcal{N}=8$ gauged supergravity, and most of them have naked singularities. These naked singularities can be regarded as artifacts of the Kaluza-Klein reduction from ten to five dimensions provided the ten-dimensional geometry avoids the aforementioned pathology of D3branes with negative tension and negative charge. As we will show in section 3.2, the pathological cases for Coulomb branch flows are precisely those where (2) is violated. 


\subsection{Finite Temperature}

The finite temperature generalization of the ansatz (1) is

$$
\begin{aligned}
d s^{2} & =e^{2 A(r)}\left(-h(r) d t^{2}+d \vec{x}^{2}\right)+\frac{d r^{2}}{h(r)} \\
\vec{\varphi} & =\vec{\varphi}(r) .
\end{aligned}
$$

Plugging (6) into the equations of motion following from (3), we obtain the following:

$$
\begin{aligned}
\square \vec{\varphi} & =h\left(\vec{\varphi}^{\prime \prime}+4 A^{\prime} \vec{\varphi}^{\prime}\right)+h^{\prime} \vec{\varphi}^{\prime}=\frac{\partial V}{\partial \vec{\varphi}} \\
G_{t}^{t}-G^{x}{ }_{x} & =-\frac{1}{2}\left(h^{\prime \prime}+4 A^{\prime} h^{\prime}\right)=0 \\
G_{t}^{t}-G_{r}^{r} & =3 h A^{\prime \prime}=-2 h \vec{\varphi}^{2}
\end{aligned}
$$

and the "zero energy" constraint

$$
G_{r}^{r}=\frac{3}{2} A^{\prime}\left(h^{\prime}+4 A^{\prime} h\right)=h \vec{\varphi}^{2}-2 V(\vec{\varphi}) .
$$

Differentiating (8) with respect to $r$ yields an equation which follows algebraically from (7), as $r$-reparametrization invariance demands.

We can solve for $A(r)$ and $h(r)$ in terms of $\vec{\varphi}^{\prime}$ using only quadratures:

$$
\begin{aligned}
& A(r)=\frac{r}{L}-\int_{r}^{\infty} d r_{1} \int_{r_{1}}^{\infty} d r_{2} \frac{2}{3} \vec{\varphi}^{\prime}\left(r_{2}\right)^{2} \\
& h(r)=1-\int_{r}^{\infty} d r_{1} \frac{4 B}{L} e^{-4 A\left(r_{1}\right)}
\end{aligned}
$$

In (9) we have specified some boundary conditions at $r \rightarrow \infty$. Choosing $\lim _{r \rightarrow \infty} h(r)=1$ near the boundary removes the freedom to rescale $r$ multiplicatively. The zero energy constraint fixes $\lim _{r \rightarrow \infty}\left(h(r) A^{\prime}(r)^{2}+\right.$ $\left.\frac{1}{3} V(\vec{\varphi}(r))\right)=0$, so if one defines $L$ through the equation $\lim _{r \rightarrow \infty} V(\vec{\varphi}(r))$ $=-3 / L^{2}$, the normalization of the linear term in $A(r)$ in (9) is fixed. We are free to add a constant to $A(r)$, or to shift $r$ itself by a constant. One of these freedoms can be absorbed by making a uniform dilation on $t$ and $\vec{x}$; the other amounts to a conformal transformation which scales all dimensionful quantities uniformly according to their dimensions. The only free integration constant in (9) with physical meaning is $B$, 
which is essentially a non-extremality parameter. If $B$ is negative, $h(r)$ would never be zero, and we would get solutions which are variants of negative mass AdS-Schwarzschild. These we regard as manifestly unphysical.

Suppose now that $B>0$. The function $h(r)$ can have at most one zero, because it is monotonic. If a zero exists, it indicates the presence of a black hole horizon. Its location, $r=r_{H}$, must satisfy $r_{H} \geq$ $\frac{L}{4} \log B$, with equality if and only if no scalars are excited (that is, for AdS-Schwarzschild only). The Hawking temperature and BekensteinHawking entropy (per unit volume in the $\vec{x}$-directions) are

$$
\begin{aligned}
T & =\frac{e^{A\left(r_{H}\right)} h^{\prime}\left(r_{H}\right)}{4 \pi}=\frac{B}{\pi L} e^{-3 A\left(r_{H}\right)} \\
\frac{S}{V} & =\pi e^{3 A\left(r_{H}\right)} .
\end{aligned}
$$

In a Euclidean version of (6), with Euclidean time $\tau=i t$, the good coordinates at $r=r_{H}$ are

$$
y=2 \sqrt{\frac{r-r_{H}}{h^{\prime}\left(r_{H}\right)}} \quad \theta=e^{A\left(r_{H}\right)} \frac{h^{\prime}\left(r_{H}\right)}{2} \tau .
$$

In terms of these variables, the $\tau-r$ part of the metric near $r=r_{H}$ is just $d y^{2}+y^{2} d \theta^{2}$. Taking the $h \rightarrow 0$ limit of the scalar equation of motion, one arrives at the following horizon boundary conditions $r=r_{H}$ :

$$
h^{\prime} \vec{\varphi}^{\prime}=\frac{\partial V}{\partial \vec{\varphi}} .
$$

The other equations of motion do not lead to any further boundary conditions in the sense of specifying a well-defined boundary value problem. The condition (12) is actually more informative than the obvious requirement that $\vec{\varphi}$ has to be smooth in the good variables (11). In those variables, (12) becomes $\frac{2}{y} \frac{d \vec{\varphi}}{d y}=\frac{\partial V}{\partial \vec{\varphi}}$.

Existence of solutions of the form (6) with regular black hole horizons does not contradict no-hair theorems. The reason is that the scalars $\vec{\varphi}$ are by assumption tachyons in the asymptotically $A d S_{5}$ region. For a particular scalar (call it $\varphi$ ) the linearized wave equation near the boundary of $A d S_{5}$ has two solutions (call them $\varphi_{(1)}$ and $\varphi_{(2)}$ ). Both of them decay as one approaches the boundary. The black hole 
horizon boundary conditions, (12), lead to $n$ constraints if there are $n$ scalars. But there are $2 n$ integration constants for the scalars, leaving us with the expectation of an $n$ parameter family of solutions.

In this section we will obtain necessary conditions for the existence of black hole solutions of the form (6). In particular, given a Poincaré invariant solution which violates (2), we will show that there does not exist a family of black hole solutions which can be made to approximate the Poincaré invariant solution arbitrarily closely. This amounts to the statement that the Poincaé invariant solution does not have near-extremal generalizations. In fact, more stringent conditions than (2) are probably necessary in order for a singular geometry to have near-extremal generalizations. Conjectures regarding what form those conditions might take will be presented in sections 3.3 and 6 .

Suppose one starts with a Poincaré invariant, singular solution to (5), which can be proven not to have near-extremal generalizations in five-dimensional classical supergravity. We wish to conclude that the dual field theory cannot support finite temperature. This is a tricky claim to make, because the curvature singularity interferes with our ability to compute reliably in classical supergravity. Two points in favor of it are as follows. 1) Assuming there is a "microscopic" description of finite temperature at a singularity which applies beyond the applicability of supergravity, there should be a smooth match from the microscopic description to the black hole horizon description at some crossover point [38]. 2) Numerical studies of two examples for which the field theory dual is reasonably well-understood indeed exhibit a smooth limit in which black hole solutions converge to a Poincaré invariant solution. We will briefly report on the numerical work in section 6 , where there is also a further discussion of the potential pitfalls of identifying solutions that can support finite temperature with those which admit near-extremal generalizations. For now we will content ourselves with an analysis of what the existence of a static black hole horizon implies.

Let us start with a regular black hole solution of the form (6). For the moment let us not assume that the solution is asymptotically $A d S_{5}$. Evaluating the zero-energy constraint (8) at the horizon $(h=0)$, we see that $A^{\prime} h^{\prime}=-\frac{4}{3} V(\vec{\varphi})$. This implies $V\left(\vec{\varphi}\left(r_{H}\right)\right)<0$. The reason is that both $A^{\prime}$ and $h^{\prime}$ are everywhere positive. In the case of $A^{\prime}$, this follows from the property $A^{\prime \prime} \leq 0$ (obvious from the third line of 
(7)), and the assumption that $A^{\prime}(r)$ is positive somewhere outside the horizon. In the case of $h^{\prime}$, it follows once one has ruled out the solutions akin to negative mass Schwarzschild: $e^{4 A} h^{\prime}$ is a constant, and to avoid negative mass it has to be a positive constant. This derivation scarcely relies on the matter lagrangian: $A^{\prime \prime} \leq 0$ is in fact implied by the null energy condition, just as in the extremal solution [18]. The conclusion, then, from the zero energy constraint, is that the bulk cosmological constant cannot be positive at the black hole horizon. The only way to saturate this bound is to take $A^{\prime}$ and $h^{\prime \prime}$ identically 0 . This gives a rather trivial solution to the vacuum Einstein equations with zero cosmological constant.

It is in fact possible to improve on the bound $V\left(\vec{\varphi}\left(r_{H}\right)\right)<0$ if one is willing to use properties of the matter lagrangian and the assumption that the spacetime is asymptotically $A d S_{5}$. Note that

$$
\begin{aligned}
\frac{d}{d r}\left(V(\vec{\varphi}(r))-\frac{1}{2} h(r) \vec{\varphi}^{\prime}(r)^{2}\right) & =\frac{\partial V}{\partial \vec{\varphi}} \vec{\varphi}^{\prime}-\frac{1}{2} h^{\prime} \vec{\varphi}^{2}-h \vec{\varphi}^{\prime} \vec{\varphi}^{\prime \prime} \\
& =\frac{1}{2}\left(h^{\prime}+8 A^{\prime} h\right) \vec{\varphi}^{\prime 2} \geq 0 \quad \text { if } h \geq 0,
\end{aligned}
$$

where to obtain the second equality we have used the scalar equation of motion. At the black hole horizon, $h=0$ and $\vec{\varphi}^{\prime}$ is finite. At the boundary of $A d S_{5}, h \rightarrow 1$ and $\vec{\varphi}^{\prime} \rightarrow 0$. So $h \vec{\varphi}^{2}=0$ both at the black hole boundary and at the horizon of $A d S_{5}$. Integrating (13), we find

$$
V\left(\vec{\varphi}\left(r_{H}\right)\right) \leq V_{U V},
$$

where $V_{U V}$ is the value of $V(\vec{\varphi})$ at the ultraviolet fixed point-usually at $\vec{\varphi}=0$. The inequality is saturated only for AdS-Schwarzschild. A crucial ingredient in obtaining these inequalities is that the scalar kinetic term is positive definite. This is implied by the null energy condition. The same positivity properties were the basis of the proofs of the c-theorem in $[14,18]$.

In view of (9), we can specify a solution to (7) completely through the pair $(\vec{\varphi}(r), B)$. Suppose we have a sequence of regular black hole solutions, specified as pairs $\left(\vec{\varphi}_{n}(r), B_{n}\right)$, with horizon radii $r_{n}$, which converges uniformly to a Poincaré invariant solution $\vec{\varphi}_{0}(r)$ to (5). More precisely, the convergence criteria are $B_{n} \rightarrow 0$ and $\sup _{r \geq r_{n}} \mid \vec{\varphi}_{n}(r)-$ $\vec{\varphi}_{0}(r) \mid \rightarrow 0$ as $n \rightarrow \infty$. Since $B_{n} \rightarrow 0$, the horizon radii $r_{n}$ will retreat as $n \rightarrow \infty$ to locations with more and more negative $A(r)$-either 
to the naked singularity of the Poincaré invariant solution, or to $r=$ $-\infty$ if there is no naked singularity. ${ }^{2}$ With minimal assumptions of smoothness on $\vec{\varphi}_{0}(r)$ and $V(\vec{\varphi})$, we can deduce from (14) that

$$
V\left(\vec{\varphi}_{0}(r)\right) \leq V_{U V} \quad \text { in the infrared. }
$$

This is now a constraint on the extremal solution.

By "in the infrared," we mean that the inequality should hold for all values of $r$ such that $A(r)$ is sufficiently negative, or at least asymptotically as $A \rightarrow-\infty$. As before, $V_{U V}$ is the limit of $V(\vec{\varphi}(r))$ as we approach the boundary of $A d S_{5}$. The condition (15) implies (2), given that $V$ is a continuous function of $\vec{\varphi}$. It is almost true that (2) implies (15): the simplest way in which one could imagine (2) holding and (15) failing is for $\vec{\varphi}$ to approach some constant value in the infrared, corresponding to an infrared fixed point in the dual field theory, such that $V_{I R}>V_{U V}$-but precisely this possibility is ruled out by the ctheorem of $[14,18]$. We will encounter several examples which violate both (2) and (15) by having $V(\vec{\varphi}(r)) \rightarrow+\infty$ in the infrared. All of these examples involve naked singularities, and these particular naked singularities seem very unphysical.

Before turning to Coulomb branch flows, a remark on different topologies on the space of field configurations is perhaps in order. Because $A(r)$ and $h(r)$ can be expressed in terms of $\vec{\varphi}(r)$ and $B$ through quadratures, it seems natural to define a norm in terms of $\vec{\varphi}$ and $B$ alone. Given two configurations, $(\vec{\varphi}, B)$ and $(\tilde{\vec{\varphi}}, \tilde{B})$, a natural Sobolev norm on the difference $\vec{\varphi}-\tilde{\vec{\varphi}}$ is

$$
\begin{aligned}
\|\vec{\varphi}-\tilde{\vec{\varphi}}\|_{H_{s}^{p}} & =\sum_{k=0}^{s}\left\|\vec{\varphi}^{(k)}-\tilde{\vec{\varphi}}^{(k)}\right\|_{L^{p}} \\
& =\sum_{k=0}^{s}\left(\int_{r_{H}}^{\infty} d r e^{4 \tilde{A}(r)}\left|\vec{\varphi}^{(k)}-\tilde{\vec{\varphi}}^{(k)}\right|^{p}\right)^{1 / p}
\end{aligned}
$$

for integer $s$. Here $\vec{\varphi}^{(k)}$ indicates the $k$-th derivative with respect to $r$, and we have assumed that the horizon radius $r_{H}$ for the configuration

\footnotetext{
${ }^{2}$ A naked singularity has $A(r) \rightarrow-\infty$ at finite $r$. If $A(r) \rightarrow-\infty$ only as $r \rightarrow-\infty$, curvatures could still grow arbitrarily large. Either way, the geometry "ends" when $A \rightarrow-\infty$, modulo issues of analytic continuation, which can be largely disregarded if we think of Wick rotating with respect to Poincaré time rather than some modification of global $A d S_{5}$ time.
} 
$(\vec{\varphi}, B)$ (defined as the location where $h=0$ ) is larger than the horizon radius $\tilde{r}_{H}$ for $(\tilde{\vec{\varphi}}, \tilde{B})$. We have chosen the measure $d r e^{4 \tilde{A}(r)}$ because it descends naturally from the five-dimensional volume element $d^{5} x \sqrt{g}$. Other measures could be considered if they seem more convenient. For $C^{\infty}$ functions, the $H_{s}^{\infty}$ norm is particularly simple:

$$
\|\vec{\varphi}-\tilde{\vec{\varphi}}\|_{H_{s}^{p}}=\sum_{k=0}^{s} \sup _{r \geq r_{H}}\left|\vec{\varphi}^{(k)}(r)-\tilde{\vec{\varphi}}^{(k)}(r)\right| .
$$

Thus the topology of uniform convergence, which we used to derive (15), follows from the $H_{0}^{\infty}$ norm, more commonly known as the $L^{\infty}$ norm. $H_{s}^{\infty}$ norms are very permissive near the boundary of $A d S_{5}$ : any relevant deformation of the lagrangian has finite norm. The $H_{s}^{2}$ norms rule out such deformations for $\mathcal{N}=4$ gauge theory, but also they rule out VEV's for dimension 2 operators. It is easy to construct norms which rule out the $r e^{-2 r / L}$ behavior of a dimension 2 deformation but permit the $e^{-2 r / L}$ behavior of a dimension $2 \mathrm{VEV}$. An example is

$$
\|\vec{\varphi}-\tilde{\vec{\varphi}}\|_{f}=\int_{2}^{\infty} d p f(p)\|\vec{\varphi}-\tilde{\vec{\varphi}}\|_{H_{s}^{p}}
$$

where $f(p)$ is a function which is 1 near $p=2$ and whose integral over $(2, \infty)$ is finite. It would be interesting to see if the topology induced by such a norm could be used in place of the topology of uniform convergence for deriving (15) as a necessary condition.

\subsection{The Coulomb branch of $\mathcal{N}=4$ super-Yang- Mills}

Although it is nice that one can start with an arbitrary black hole solution and prove definite statements like (14) that support the view that the scalar potential must remain bounded above near a curvature singularity, one may still retain some doubt about the validity of (2). In particular, might it not be that singularities which violate (2) simply correspond to vacua in the dual field theory which cannot support finite temperature? In this section, we will argue against this possibility by considering explicit examples of such vacua where the dual geometries are well understood, singular, and in line with (2). 
Next to the maximally supersymmetric $A d S_{5}$ geometry, the bestunderstood solutions to $d=5 \mathcal{N}=8$ gauged supergravity with $3+1$ dimensional Poincaré invariance are those which involve only the metric and the scalars in the coset $S L(6, \mathbf{R}) / S O(6)[6,8,9,36,10,21]$. The CFT duals (when they are well-defined) are states on the Coulomb branch of $\mathcal{N}=4$ super-Yang-Mills which can be parametrized by the VEV's of operators $\operatorname{tr} X_{(I} X_{J)}$. These operators, transforming in the $\mathbf{2 0}$ of $S O(6)$, are precisely the ones dual to the 20 scalars parametrizing $S L(6, \mathbf{R}) / S O(6)$.

There are VEV's for higher dimension operators as well as for those in the $\mathbf{2 0}^{\prime}$, and one can calculate them systematically from the tendimensional geometry. The magic of consistent truncation is that the profiles of only finitely many scalars capture infinitely many VEV's. Roughly speaking, these higher dimension VEV's are sourced by arbitrary powers of the scalars in the $\mathbf{2 0}^{\prime}$. The corresponding statement in consistent truncation is that the ten-dimensional fields are determined non-linearly in terms of the five-dimensional fields.

The ten-dimensional geometry is the near-horizon limit of the background of a continuous distribution of D3-branes:

$$
\begin{aligned}
d s^{2} & =\frac{1}{\sqrt{H}}\left(-d t^{2}+d \vec{x}^{2}\right)+\sqrt{H} \sum_{I=1}^{6} d y_{I}^{2} \\
H & =\int d^{6} \xi \sigma(\vec{\xi}) \frac{L^{4}}{|\vec{y}-\vec{\xi}|^{4}},
\end{aligned}
$$

where the distribution $\sigma$ integrates to 1 . All the solutions whose KaluzaKlein reductions involve only the five-dimensional metric and the scalars in $S L(6, \mathbf{R}) / S O(6)$ arise from distributions which are limits of either of the following the following two distributions [10]:

$$
\begin{aligned}
\sigma_{6}(\vec{\xi})= & \frac{1}{\pi^{3} \ell_{1} \cdots \ell_{6}} \delta^{\prime}\left(1-\sum_{I=1}^{6} \frac{\xi_{I}^{2}}{\ell_{I}^{2}}\right) \\
\sigma_{5}(\vec{\xi})= & \frac{1}{2 \pi^{3} \ell_{1} \cdots \ell_{5}}\left[-\left(1-\sum_{I=1}^{5} \frac{\xi_{I}^{2}}{\ell_{I}^{2}}\right)^{-3 / 2} \Theta\left(1-\sum_{I=1}^{5} \frac{\xi_{I}^{2}}{\ell_{I}^{2}}\right)\right. \\
& \left.+2\left(1-\sum_{I=1}^{5} \frac{\xi_{I}^{2}}{\ell_{I}^{2}}\right)^{-1 / 2} \delta\left(1-\sum_{I=1}^{5} \frac{\xi_{I}^{2}}{\ell_{I}^{2}}\right)\right] \delta\left(\xi_{6}\right) .
\end{aligned}
$$


The distribution $\sigma_{5}(\vec{\xi})$ is the limit of $\sigma_{6}(\vec{\xi})$ as $\ell_{6} \rightarrow 0$. There is a sort of shell theorem to the effect that shifting all the $\ell_{I}^{2}$ by a constant makes no difference to the geometry away from the branes (see $[6,24,25]$ for evidence of this theorem, and [10] for a crisp statement). Both $\sigma_{5}$ and $\sigma_{6}$ fail to be everywhere positive. This is the signal that there are "ghost" D3-branes of negative tension and negative charge, as well as normal D3-branes with positive tension and positive charge. In the absence of an understanding of the geometries involving "ghosts" along the lines of [37], we are inclined to regard them as unphysical. If we send $\ell_{5} \rightarrow 0$ in $\sigma_{5}$, the resulting distribution is a delta function shell in the shape of an ellipsoid:

$$
\sigma_{4}(\vec{\xi})=\frac{1}{\pi^{2} \ell_{1} \cdots \ell_{4}} \delta\left(1-\sum_{I=1}^{4} \frac{\xi_{I}^{2}}{\ell_{I}^{2}}\right) \delta\left(\xi_{5}\right) \delta\left(\xi_{6}\right) .
$$

This is a positive definite distribution, and so are its limits with one or more of the remaining $\ell_{I}$ taken to zero.

Given a distribution $\sigma(\vec{\xi})$, vacuum expectation values of gauge singlet operators can be computed by the rule

$$
\left\langle\operatorname{tr} X_{\left(I_{1}\right.} \cdots X_{\left.I_{\ell}\right)}\right\rangle=\int d^{6} \xi \sigma(\vec{\xi}) \xi_{I_{1}} \cdots \xi_{I_{\ell}} .
$$

Conversely, a distribution of the form (20) (or any limit thereof) can be reconstructed from the VEV's of the $S O(6)$ irrep operators $\operatorname{tr} X_{\left(I_{1}\right.} \cdots X_{\left.I_{\ell}\right)}$, up to an ambiguity which amounts to shifting all the $\ell_{I}^{2}$ by a constant. Thus the requirement that the distribution can be made positive definite (by appropriate choice of this constant shift) must translate into a series of inequalities among the VEV's for $\operatorname{tr} X_{\left(I_{1}\right.} \cdots X_{\left.I_{\ell}\right)}$ which follow from the hermiticity of the individual $X_{I}$. The point of all this is that the "ghost" D3-branes are in this case really unphysical: no local resolution of the geometry which left the far field form unchanged would change the problematic VEV's.

Let us first take $n$ of the $\ell_{I}$ equal in (20), and set the other $6-n$ to zero. These cases were studied in detail in [8]. The $S O(6)$ global symmetry is broken to $S O(n) \times S O(6-n)$. The five-dimensional geometry involves only one scalar. More precisely, the trajectory on the scalar manifold is a geodesic with respect to the sigma-model metric, and we can parametrize it with a canonically normalized scalar, $\mu$. The 
operator dual to $\mu$ is $\mathcal{O}_{2}=\operatorname{tr}\left[(6-n) \sum_{I \leq n} X_{I}^{2}-n \sum_{I>n} X_{I}^{2}\right]$. Near the boundary of $A d S_{5}$, we have $\mu \sim e^{-2 r / L}$, corresponding to a positive VEV for $\mathcal{O}_{2}$. Far from the boundary, $\mu$ increases without bound. For large $\mu$, one finds $W(\mu) \sim-e^{\zeta \mu}$. The constant $\zeta$ has values $2 / \sqrt{15}$, $\sqrt{2 / 3}, \sqrt{4 / 3}, \sqrt{8 / 3}$, and $10 / \sqrt{15}$ for $n=1,2,3,4$, and 5 respectively (see (15) of [8]). The geometric origin of these numbers will become clear shortly.

For $n \leq 4$ one finds $V(\mu) \rightarrow-\infty$ as $\mu \rightarrow \infty$, whereas for $n=5$ one finds $V(\mu) \rightarrow+\infty$. Thus (2) rules out the pathological $n=5$ case but accepts the physical $n=4$ cases. This success, which seems to have nothing to do with finite temperature, is our second motivation for proposing (2) as a good criterion for distinguishing physical from unphysical geometries.

It is fairly straightforward $[36,10]$ to work out the cases with unequal $\ell_{I}$. Five scalars are involved in general, corresponding to the five independent eigenvalues of the matrix $M=S S^{T}$, where $S \in$ $S L(6, \mathbf{R}) / S O(6)$. In an appropriate basis, $M=\operatorname{diag}\left\{e^{2 \beta_{1}}, e^{2 \beta_{2}}, \ldots\right.$, $\left.e^{2 \beta_{6}}\right\}$, and $\sum_{k} \beta_{k}=0$. Following [8], we introduce scalars $\varphi_{1}$ through $\varphi_{5}$ via the relation

$$
\left(\begin{array}{l}
\beta_{1} \\
\beta_{2} \\
\beta_{3} \\
\beta_{4} \\
\beta_{5} \\
\beta_{6}
\end{array}\right)=\left(\begin{array}{ccccc}
1 / \sqrt{2} & 1 / \sqrt{2} & 1 / \sqrt{2} & 0 & 1 / \sqrt{6} \\
1 / \sqrt{2} & -1 / \sqrt{2} & -1 / \sqrt{2} & 0 & 1 / \sqrt{6} \\
-1 / \sqrt{2} & -1 / \sqrt{2} & 1 / \sqrt{2} & 0 & 1 / \sqrt{6} \\
-1 / \sqrt{2} & 1 / \sqrt{2} & -1 / \sqrt{2} & 0 & 1 / \sqrt{6} \\
0 & 0 & 0 & 1 & -\sqrt{2 / 3} \\
0 & 0 & 0 & -1 & -\sqrt{2 / 3}
\end{array}\right)\left(\begin{array}{l}
\varphi_{1} \\
\varphi_{2} \\
\varphi_{3} \\
\varphi_{4} \\
\varphi_{5}
\end{array}\right) .
$$

The sigma model metric on the five-dimensional space parametrized by the $\varphi_{I}$ is $\delta_{I J}$. The potential, $V=-\frac{1}{8 L^{2}}\left[(\operatorname{tr} M)^{2}-2 \operatorname{tr} M^{2}\right]$, and the superpotential, $W=-\frac{1}{4} \operatorname{tr} M$, can be written explicitly as sums of exponentials:

$$
\begin{aligned}
V(\vec{\varphi}) & =\sum_{\alpha} v_{\alpha} e^{\vec{\eta}_{\alpha} \cdot \vec{\varphi}} \\
W(\vec{\varphi}) & =\sum_{\alpha} w_{\alpha} e^{\vec{\zeta}_{\alpha} \cdot \vec{\varphi}} .
\end{aligned}
$$

All the constants $w_{\alpha}$ are negative, but the same is not true of the $v_{\alpha}$. The six vectors $\vec{\zeta}_{\alpha}$ are of equal length, and they sum to zero. The level sets of $W(\vec{\varphi})$ for large $|\vec{\varphi}|$ are asymptotic to concentric "hexahedra" in $\mathbf{R}^{5}$. A hexahedron is the six-sided polyhedron which is the 
five-dimensional generalization of the tetrahedron. It has $\left(\begin{array}{l}6 \\ n\end{array}\right)(n-1)$ dimensional "faces," where a zero-dimensional "face" is a vertex, a one-dimensional "face" is an edge, and so on up to $n=6$. The fourdimensional faces of the hexahedra are normal to the $\vec{\zeta}_{\alpha}$. The geodesic gradient flow trajectories which preserve $S O(n) \times S O(6-n)$ symmetry are straight lines in $\mathbf{R}^{5}$ which pass through the center of an $n-1$ dimensional face of each hexahedron; alternatively we may describe them as the rays emanating from the origin of $\mathbf{R}^{5}$ parallel to a sum of $6-n$ of the vectors $\vec{\zeta}_{\alpha}$. Other gradient trajectories of $W(\vec{\varphi})$, which lift to ten-dimensional geometries with unequal $\ell_{I}$, must run asymptotically parallel to one of the geodesics we have described. Those which run asymptotically parallel to some $\vec{\zeta}_{\alpha}$ correspond to cases with five non-zero $\ell_{I}$, and these are the ones we wish to rule out on account of the pathology of "ghost" D3-branes. This is exactly what the condition (2) does. The region $V(\vec{\varphi})<-3 / L^{2}$ includes all the faces of the hexahedra of dimension less than four, but it excludes more and more of the four-dimensional faces as $|\vec{\varphi}|$ increases, so that asymptotically none of the trajectories perpendicular to these faces are allowed.

All this may be easier to visualize by extension from figure 1 , which shows a two-dimensional analog with three-fold symmetry to the fivedimensional example (24) with six-fold symmetry.

\subsection{Asymptotics and phase transitions}

In all of the examples in section 4 , we will find $V(\vec{\varphi})$ and $W(\vec{\varphi})$ with the same form as (24), but in some cases some of the $w_{\alpha}$ will be positive. A gradient flow trajectory of $W$ which does not end at a saddle point will have an asymptotic direction, which we may specify by a unit vector $\vec{n}$. Let us parametrize the trajectory by a canonically normalized scalar $\mu$. From (5) one can extract a necessary condition on $\vec{n}$, namely

$$
-\sum_{\alpha} w_{\alpha} \vec{\zeta}_{\alpha} e^{\vec{\zeta}_{\alpha} \cdot \mu \vec{n}} \| \vec{n} \quad \text { asymptotically as } \mu \rightarrow \infty,
$$

where $\vec{u} \| \vec{v}$ means that $\vec{u}$ is parallel to $\vec{v}$ : one is a positive multiple of the other. There is a straightforward geometrical construction to identify the solutions to (25). For each vector $\vec{\zeta}_{\alpha}$, define $H_{\alpha}=\{\vec{\varphi}$ : $\left.\vec{\varphi} \cdot \vec{\zeta}_{\alpha}<1\right\}$. The intersection $H=\bigcap_{\alpha} H_{\alpha}$ is convex. It is a compact 


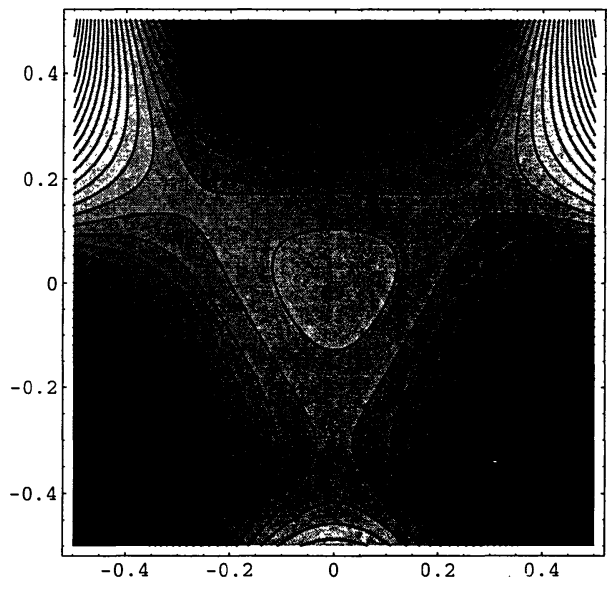

(a)

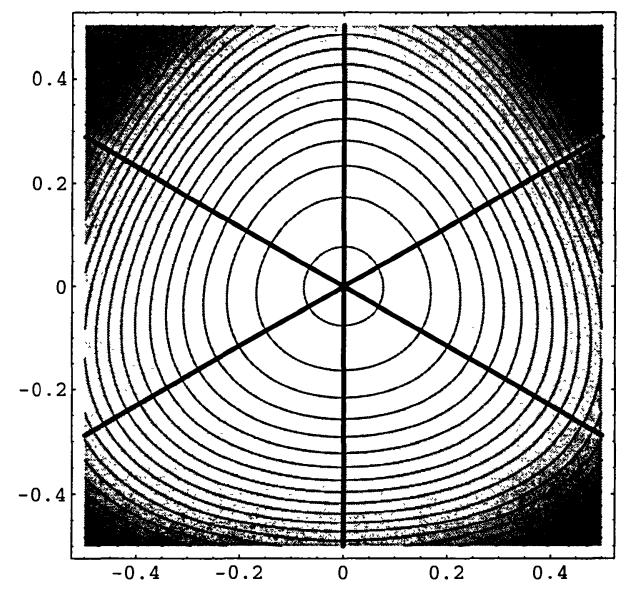

(b)

Figure 1: (a) Contour plot of $V$ and (b) contour plot of $W$, for $W(\vec{\varphi})=$ $-\frac{1}{4} e^{\sqrt{3} \varphi_{1}+\varphi_{2}}-\frac{1}{4} e^{-\sqrt{3} \varphi_{1}+\varphi_{2}}-\frac{1}{4} e^{-2 \varphi_{2}}$ and $V(\vec{\varphi})=\frac{1}{8}\left(\frac{\partial W}{\partial \vec{\varphi}}\right)^{2}-\frac{1}{3} W(\vec{\varphi})^{2} \cdot W$ was chosen arbitrarily, but $V$ and $W$ exhibit the same features in two dimensions that obtain in five for the $V(\vec{\varphi})$ and $W(\vec{\varphi})$ that describe Coulomb branch states for $\mathcal{N}=4$ super-Yang-Mills. Regions of higher elevation are lighter in shade. If the region of the plots were much larger, one would see that $V(\vec{\varphi}) \rightarrow+\infty$ as $|\vec{\varphi}| \rightarrow \infty$, unless one proceeds in one of three special directions separated by $120^{\circ}$ : north, east-southeast or west-southwest. The center of both plots is the local maximum representing the maximally supersymmetric $A d S_{5}$ vacuum. The three saddle points of $V(\vec{\varphi})$ are analogs of the unstable $S O(5)$ critical points. The thick lines in (b) represent the set $\mathcal{P}$, to be defined in (28). This figure, as well as figures 4,5 , and 6 , may be easiest to read in color. 
polyhedron in all examples we will encounter. Mathematically, it is similar in construction to a Brillouin zone, only the vectors $\vec{\zeta}_{\alpha}$ do not form a lattice. Now define a function $\sigma: \partial H \rightarrow\{-1,0,+1\}$ as follows. Given a point $P$ on the boundary $\partial H$ of $H$, consider all those $H_{\alpha}$ for which $P$ is also on the boundary of $H_{\alpha}$. If all the associated $w_{\alpha}$ are negative, let $\sigma(P)=-1$; if all are positive, let $\sigma(P)=+1$; and if some are positive and some are negative, let $\sigma(P)=0$. Define an apothem of $H$ as a vector with its tail at the origin and its head on $\partial H$, such that the vector is orthogonal to the lowest-dimensional face of $H$ which it touches. We are again thinking of faces in the generalized sense: they can be vertices, edges, two-dimensional faces, etc. The apothems of $H$ include the vectors $\vec{\zeta}_{\alpha} /\left|\vec{\zeta}_{\alpha}\right|^{2}$, and also certain linear combinations of these vectors. If $\vec{\kappa}$ is an apothem of $H$ such that $\sigma=-1$ at the head of the apothem, then there is a solution $\vec{n}$ to (25) which is parallel to $\vec{\kappa}$. And any solution $\vec{n}$ to (25) must be parallel to an apothem of $H$ for which $\sigma=-1$ or 0 . Along a gradient flow trajectory which is asymptotically parallel to an apothem $\vec{\kappa}$, we will have $W \sim-e^{\zeta \mu}$ for large $\mu$, where $\zeta=1 /|\vec{\kappa}|$. Since

$$
V \approx \frac{1}{8}\left(\frac{\partial W}{\partial \mu}\right)^{2}-\frac{1}{3} W^{2},
$$

we learn that $V \rightarrow-\infty$ if $\zeta<\sqrt{8 / 3}$, and $V \rightarrow+\infty$ if $\zeta>\sqrt{8 / 3}$. Thus the condition (2) rules out directions in which $|\vec{\kappa}|<\sqrt{3 / 8}$. In the cases where $\sigma=0$ at the head of $\vec{\kappa}$ or where $|\vec{\kappa}|=\sqrt{3 / 8}$, the behaviors of $W$ and $V$ have to be checked explicitly.

If we imagine $W(\vec{\varphi})$ as a mountain, and ourselves as skiers, the vectors $\vec{\kappa}$ indicate the possible asymptotic directions that we could take if we started at $\vec{\varphi}=0$ and followed the fall line all the way down. Vectors with $|\vec{\kappa}|<\sqrt{3 / 8}$ indicate directions which are "too steep" for safe skiing and violate $(2)$ as a result. When all the $w_{\alpha}$ are negative, the level curves of $W(\vec{\varphi})$ for large $|\vec{\varphi}|$ approximate concentric dilated copies of $\partial H$. When some of the $w_{\alpha}$ are positive and some are negative, the level sets are more complicated, but for large negative or large positive values of $W$ they include the faces of dilations of $\partial H$ with $\sigma=-1$ or $\sigma=+1$, respectively.

Just as we constructed the convex polyhedron $H$ and the sign map $\sigma$ starting with $W$ in the form of a sum of exponentials, we can construct a convex polyhedron $K$ and a sign map $\rho$ starting with $V$. Let $L$ be 
the union of rays which start at the origin and pass through a point on $\partial K$ where $\rho=0$. The codimension of $L$ is at least one. The zero contour of $V$ must be asymptotic to $L$ for large $|\vec{\varphi}|$. It is not guaranteed that every ray in $L$ is asymptotically close to the zero contour of $V$, but this is guaranteed for a ray in $L$ associated with a point $P$ on $\partial K$ such $\rho$ takes both positive and negative values close to $P$. The set $L$ divides the space of scalars into wedges which do or do not violate (2) according as $\rho=+1$ or $\rho=-1$ for the face(s) of $K$ lying in that wedge. Gradient flow trajectories which run on or asymptotic to $L$ must be checked explicitly.

It is transparent from the foregoing discussion that trajectories which pass (2) for potentials of the form (24) will also satisfy

$$
\frac{\partial W}{\partial \vec{\varphi}} \| \frac{\partial V}{\partial \vec{\varphi}} \quad \text { asymptotically as } A(r) \rightarrow-\infty .
$$

This condition can also be motivated (although not rigorously) by finite temperature considerations. In the far region of a near-extremal geometry, where $h \approx 1$, the equations (7) are approximately solved by a solution to the first order equations, (5). In the near-horizon region, (12) should apply to a good approximation. It is natural to think that the far and near regions can be matched onto each other provided $\vec{\varphi}^{\prime}$ is pointing roughly in the same direction in both regions. Modulo some (fairly strong) technical assumptions, it is possible to show that (27) is a necessary condition for the existence of a sequence of nonextremal solutions which converge to an extremal solution in the $H_{1}^{\infty}$ norm. However, the $H_{1}^{\infty}$ norm is a very strong topology in the infrared, and it may be that near-extremal solutions do not in general converge to an extremal limit in this norm.

In exploring examples, it is often convenient to look first at a more restricted category of trajectories than (2) allows. Let us define

$$
\mathcal{P}=\left\{\vec{\varphi}: \pm \frac{\partial W}{\partial \vec{\varphi}} \| \frac{\partial V}{\partial \vec{\varphi}}\right\} .
$$

Given $W$ and $V$ in closed form, it is particularly straightforward to find $\mathcal{P}$ because it is the solution set to the equations

$$
\epsilon^{i_{1} \cdots i_{n}}\left(\partial W / \partial \varphi^{i_{1}}\right)\left(\partial V / \partial \varphi^{i_{2}}\right)=0
$$


Generically, $\mathcal{P}$ consists of several intersecting curves. Some of them are asymptotic to gradient flow trajectories of $W$ in the limit where $A \rightarrow-\infty$. They can sometimes be excellent approximations to particular gradient flow trajectories even at finite $A$. In the Coulomb branch example of section $3.2, \mathcal{P}$ consists of lines through the origin passing perpendicularly through every face (of every dimension) of the hexahedron. It is thus precisely the union of all the gradient flow trajectories preserving some $S O(n) \times S O(6-n)$ symmetry. Every trajectory runs asymptotically parallel to some line in $\mathcal{P}$ in this case. $\mathcal{P}$ will not have quite such nice properties in the examples of section 4 , but it remains true (as will be seen case by case) that every trajectory which passes the criterion (2) is also asymptotically parallel to some curve in $\mathcal{P}$. It would be nice to know if there is any deep reason for this.

It is straightforward to categorize the possible behaviors in the infrared, provided the scalars' evolution has an asymptotic direction. As explained above, this assumption is the generic expectation given a potential $V(\vec{\varphi})$ of the form (4). Let us therefore consider in more detail the choice $W(\mu)=-e^{\zeta \mu}$. The variable $\mu$ is understood as the canonically normalized scalar along the asymptotic direction of the flow. Our only aim is to obtain the correct scaling behavior, so we drop overall factors, including dimensionful ones like the asymptotic radius of curvature of $A d S_{5}$. From (5) one easily obtains

$$
\begin{aligned}
d s^{2} & =\left(r-r_{0}\right)^{\frac{4}{3 \zeta^{2}}}\left(-d t^{2}+d \vec{x}^{2}\right)+d r^{2} \\
e^{-\zeta \mu(r)} & =\frac{\zeta^{2}}{2}\left(r-r_{0}\right),
\end{aligned}
$$

where $r_{0}$ is an integration constant which we readily identify as the location of the singularity. Curvature invariants involving $n$ derivatives diverge like $\left(r-r_{0}\right)^{-2 n}$ as $r \rightarrow r_{0}$. The causal structure of the geometry (29) depends on the value of $\zeta$, and is more transparent if one introduces a new variable $z$ such that $d r=-e^{A} d z$. Let us assume that the geometry (29) merges into asymptotically anti-de Sitter spacetime at large $r$. Then it is straightforward to show that if $\zeta>\sqrt{2 / 3}$ the Penrose diagram is a strip, as in figure 2(a), while if $\zeta \leq \sqrt{2 / 3}$ the Penrose diagram is a wedge, as in figure $2(\mathrm{~b})$. Clearly the singularity gets worse as $\zeta$ gets larger: a naked timelike singularity, as in figure $2(\mathrm{a})$, is more problematic than a null singularity of the type in figure $2(\mathrm{~b})$, which is similar to the singular horizon of the D2-brane. 


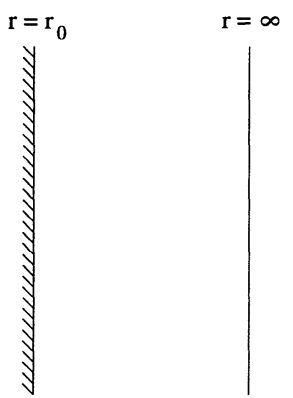

a)

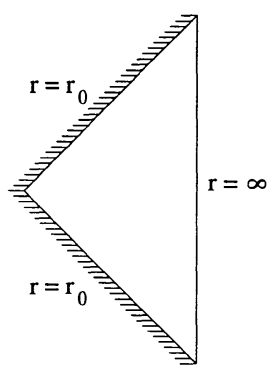

b)

Figure 2: (a) The Penrose diagram for $\zeta>\sqrt{2 / 3}$ is a strip. Curvatures diverge as one approaches the right edge. This is a naked timelike singularity. (b) The Penrose diagram for $\zeta \leq \sqrt{2 / 3}$ is a wedge. Curvatures diverge as one approaches either of the null edges. This is a null singularity.

When $\zeta>\sqrt{8 / 3}, V \rightarrow+\infty$ as $r \rightarrow r_{0}$, and the conjecture (2) is that the singularity is unphysical. Certainly it fails to admit near-extremal generalizations. When $\zeta<\sqrt{8 / 3}, V \rightarrow-\infty$, and (2) is satisfied. In this case, $|V(\mu)|$ grows slower than $e^{\sqrt{32 / 3} \mu}$. Of the solutions $W(\mu)$ to

$$
\frac{1}{8}\left(\frac{\partial W}{\partial \mu}\right)^{2}-\frac{1}{3} W^{2}=V=\left(\frac{\zeta^{2}}{8}-\frac{1}{3}\right) e^{2 \zeta \mu},
$$

only $W(\mu)= \pm e^{\zeta \mu}$ grows more slowly than $e^{\sqrt{8 / 3} \mu}$ as $\mu \rightarrow \infty$. All the other solutions are asymptotically of the form $W(\mu) \sim($ const $) e^{\sqrt{8 / 3} \mu}$. It is possible to integrate (30) analytically, but the result is an complicated implicit equation relating $W$ and $\mu$. However the asymptotics is obvious provided $|V(\mu)|$ grows slower than $e^{\sqrt{32 / 3} \mu}$. This implies that the generic Poincaré-invariant solution to the equations of motion following from the action (3) is of the form (29) with $\zeta=\sqrt{8 / 3}$-provided $|V(\vec{\varphi})|$ grows slower than $e^{\sqrt{32 / 3}|\vec{\varphi}|}$. This result has been obtained previously by a direct analysis of the equations of motion [20].

We have already demonstrated in two different ways that (2) is a necessary condition for the existence of near-extremal solutions, once by using the zero energy constraint plus the null energy condition, and once by using the scalar equation of motion. It will be instructive to present one more proof-inferior to the previous two because it is 
less rigorous and less general, but suggestive of interesting physics at finite temperature. Start with a Poincaré invariant solution generated using a $W$ which is asymptotic to $-e^{\zeta \mu}$ with $\zeta>\sqrt{8 / 3}$. To obtain near-extremal generalizations, one would solve (7) and (8) with a small value of $B$ in (9). Let us proceed on the assumption that for small $B$, the solution for $A(r)$ and $\vec{\varphi}(r)$ changes only slightly. Setting $L=1$ and neglecting factors of order unity, the expression for $h(r)$ is

$$
\begin{aligned}
h(r) & =1-B \int_{r} d r_{1} e^{-4 A\left(r_{1}\right)} \\
& \approx 1-B \int_{r} d r_{1}\left(r_{1}-r_{0}\right)^{-\frac{8}{3 \zeta^{2}}} .
\end{aligned}
$$

The integral in the second line remains finite as $r \rightarrow r_{0}$, so it is indeed true that small $B$ uniformly suppresses the effects of $h$ in the equations of motion. Thus the approximation used in the second line of (31) is reliable for sufficiently small $B$. The conclusion is that $h(r)$ has no zero for small $B$ : there is no horizon! This indeed indicates that there are no near-extremal generalizations. For sufficiently large $B$, and assuming the criterion (14) can be met, it is plausible that solutions with horizons will exist, and that they have a temperature which diverges as $B \rightarrow \infty$. Such solutions might be dubbed "far-extremal," since they are not close to the original Poincaré invariant solution. Assuming the spacetime is asymptotically $A d S_{5}$, solutions sufficiently far from extremality (in the sense of $B$ being large) would be nearly AdS-Schwarzschild. Let $B_{0}$ be the infimum of the set of $B$ 's for which there is a horizon. It is again plausible that $e^{2 A(r)}$ and $h(r)$ vanish simultaneously when $B=B_{0}$, and that for $B$ larger than $B_{0}$ but sufficiently close to it, $h(r)=0$ at a location $r=r_{H}$ where $e^{2 A(r)}$ can be made as small as we wish. Since $B_{0}$ is fixed and finite, the formula $T=\frac{B}{\pi L} e^{-3 A\left(r_{H}\right)}$ implies that the temperature diverges as $B \rightarrow B_{0}$. Thus there must be some $B_{c}>B_{0}$ where $T$ attains its minimum. (Let us assume for simplicity that $T$ as a function of $B$ has only one extremum). The mass above extremality is related monotonically to $B$. Generically one expects that this relation is approximately linear near $B=B_{c}$, and that $T \sim T_{c}+\left(B-B_{c}\right)^{2}$. Then as we approach the critical point from above (both in energy and temperature), $E-E_{c} \sim\left(T-T_{c}\right)^{1 / 2}$, which implies a divergence in the specific heat, $C \sim\left(T-T_{c}\right)^{-1 / 2}$. This is the signal of a second order phase transition at finite temperature. On the field theory side, if one makes the generic assumption that the inverse square of the correlation length goes to zero like $T-T_{c}$ (which is to say, $\nu=1 / 2$ ), then the 
specific heat exponent $\alpha=1 / 2$ emerges from the hyperscaling relation, $\alpha=2-d \nu$ with $d=3$. Why the strong interactions should not change the story drastically is unclear.

If $\zeta<\sqrt{8 / 3}$, the integral in the second line of (31) diverges as $r \rightarrow r_{0}$, and one might hope to argue on this basis that near-extremal generalizations of the Poincare invariant solution do exist. But when $h$ deviates significantly from 1 , it is hard to justify the approximation of keeping the same $A(r)$ and $\vec{\varphi}(r)$ as in the Poincaré invariant solution. Thus we cannot make any rigorous claims. Let us nevertheless attempt to obtain an equation of state, assuming the asymptotics

$$
\begin{aligned}
& A(r) \sim \alpha_{1} \log \left(r-r_{0}\right)+A_{0} \\
& h(r) \sim 1-B \int_{r} d r_{1} e^{-4 A\left(r_{1}\right)},
\end{aligned}
$$

with $\alpha_{1}<1 / 4$. Again we set $L=1$ and neglect factors of order unity, which is $\mathrm{OK}$ since the goal is only to obtain a scaling relation. Equation (10) may be used to obtain

$$
\begin{aligned}
B & =e^{4 A_{0}}\left(4 \alpha_{1}-1\right)\left(r_{H}-r_{0}\right)^{4 \alpha_{1}-1} \\
T & =e^{A_{0}}\left(4 \alpha_{1}-1\right)\left(r_{H}-r_{0}\right)^{\alpha_{1}-1} \\
\frac{S}{V} & =e^{3 A_{0}}\left(r_{H}-r_{0}\right)^{3 \alpha_{1}}
\end{aligned}
$$

The factors of $e^{A_{0}}$ indicate the how each quantity scales under a conformal transformation. $B$ indeed scales as energy density. Eliminating $r_{H}-r_{0}$ leads to

$$
\frac{S}{V} \sim T^{\eta} \quad \text { with } \quad \eta=\frac{3 \alpha_{1}}{\alpha_{1}-1}=\frac{6}{2-3 \zeta^{2}}
$$

where in the last line we have assumed that $\alpha_{1}=\frac{2}{3 \zeta^{2}}$ as in the Poincaré invariant flow, (29). The calculation is probably trustworthy when $\zeta$ is sufficiently small. Note that as $\zeta \rightarrow 0$ one recovers the scaling $S \sim T^{3}$ which applies to a conformal field theory. It is interesting to note that in the range where (34) predicts positive specific heats, it also predicts that $-F / T^{4}$ is an increasing function of temperature. This is consistent with the Appelquist-Cohen-Schmaltz conjecture [39]. ${ }^{3}$ For $\zeta>\sqrt{2 / 3}$,

\footnotetext{
${ }^{3}$ Despite efforts by the current author and by R. Myers, there is no general proof of the monotonicity of $-F / T^{4}$ in solutions of the form (1). I thank R. Myers for an extensive correspondence on this topic.
} 

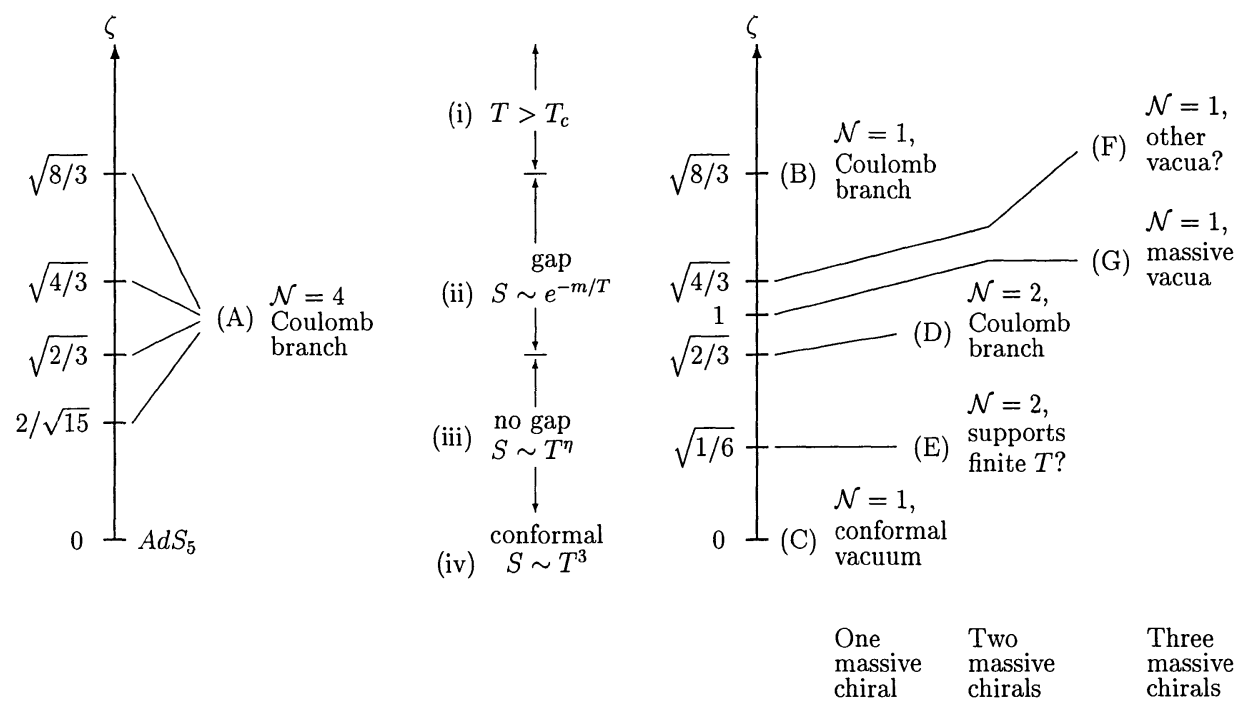

Figure 3: Far left: Coulomb branch states exhibit a wide range of $\zeta$. Middle: various expectations for the response of singularities to finite temperature. Right: a summary of the examples in section 4. Details can be found there regarding each entry.

the evidence of (34) is that $T \rightarrow \infty$ as $r_{H} \rightarrow r_{0}$. It could be that instead $T$ remains finite in this limit. Either of these two outcomes would signal a finite temperature phase transition. Or it is possible that $T \rightarrow 0$ as $r_{H} \rightarrow r_{0}$, and $S$ as a function of $T$ falls off faster than any power, indicating a mass gap.

It would be very interesting to find sufficient conditions for the existence of black hole solutions arbitrarily close to a given Poincaré invariant flow in an appropriately weak topology. It would also be interesting to find more stringent necessary conditions than (2). For instance, one might conjecture that a necessary condition for a gradient flow to have finite temperature generalizations which converge to the original geometry in the topology induced by the norm (18) is that the gradient flow should either terminate at a saddle point or converge to a curve in $\mathcal{P}$.

Section 4 is concerned with additional examples of Poincaré invariant flows where the field theory physics is more or less clear. It is useful to categorize the flows according to the value of $\zeta$ which controls their far infrared behavior. This is done in figure 3 . 
The responses to finite temperature indicated in figure 3 are based partly on calculation, partly on AdS/CFT examples where the field theory physics is clear, and partly on conjecture. The arguments (31) consitute fairly good evidence that for flows dominated by some direction in which $\zeta>\sqrt{8 / 3}$, there is a finite temperature phase transition. On the other hand, the AdS-Schwarzschild solution demonstrates that there are near-extremal geometries with arbitrarily small temperature when $\zeta=0$. The analysis leading to (34) suggests that for $\zeta<\sqrt{2 / 3}$ there are still near-extremal geometries with arbitrarily small temperature. It was found in [8] that minimally coupled scalars exhibit no gap for geometries of the form (1) when $\zeta<\sqrt{2 / 3}$. This fits with the power law behavior $S \sim T^{\eta}$ found in (34). Note that $\zeta=\sqrt{2 / 3}$ is also the value where the Penrose diagram for the Poincaré invariant flow changes from figure 2(b) to figure 2(a): for $\zeta<\sqrt{2 / 3}$ the singularity is null, while for $\zeta>\sqrt{2 / 3}$ it is timelike. What happens for $\sqrt{2 / 3}<\zeta<\sqrt{8 / 3}$ is harder to understand. Minimal scalars exhibit a mass gap, so one must expect that $S$ falls off faster than any power of $T$ for sufficiently small $T$. It could be however that curvatures at the horizon become Planckian before this behavior can be observed. If so, the conclusion is simply that a string theory resolution of the singularity is necessary before the low-temperature physics can be fully understood.

The range of $\zeta$ 's for the Coulomb branch is puzzling. How can this simple system exhibit such a range of behaviors? One possible explanation was proposed in [8]. It is that the supergravity geometries specify only a continuous distribution of D3-branes in ten dimensions, and at finite $N$ the physics is most likely an ensemble average of all discrete distributions which sufficiently closely approximate the continuous one. Roughly speaking, any D3-brane can wiggle as far as its nearest neighbor. Performing the ensemble average first in the path integral could lead to extra terms in an effective lagrangian, for instance terms of the form $\left(\Sigma^{I J} \operatorname{tr} X_{(I} X_{J)}\right)^{2}$ where $\Sigma^{I J}$ is some tensor in the $\mathbf{2 0}^{\prime}$. These extra effective interactions get larger as the dimensionality of the brane distribution increases, and could perhaps explain the peculiar properties that supergravity seems to ascribe to these configurations. A naive estimate of the strength of the double trace terms relative to the original lagrangian is $N^{1-2 / p}$ where $p$ is the dimensionality of the distribution. Another possibility (also speculative) is that the low-energy theory of open strings on a $p$-dimensional continuous distribution of D3-branes 
is effectively $p+4$ dimensional, simply because the open strings are allowed to end on any D3-brane. Then the presence or absence of a mass gap can be understood in terms of the higher dimensional theory. ${ }^{4}$

\section{Examples}

The examples of sections 4.1 and 4.3 have appeared previously in the literature $[18,20]$; the example in section 4.2 is based on work with K. Pilch and N. Warner [40]. On the supergravity side, the superpotential $W$ is associated with supersymmetry transformations: the first order equations (5) are precisely the conditions for some fraction of $d=5 \mathcal{N}=8$ supersymmetry to remain unbroken. As explained after (4), $W$ is an eigenvalue of a $S O(6) \times S L(2, \mathbf{R})$-invariant matrix $W_{a b}$. Which eigenvalue is the correct one to describe a given relevant deformation can usually be deduced from how much supersymmetry is unbroken, plus known asymptotics near the boundary of $A d S_{5}$.

On the field theory side, all the examples come from relevant deformations of $\mathcal{N}=4$ supersymmetric Yang-Mills theory which preserve at least $\mathcal{N}=1$ supersymmetry. The on-shell spectrum of $\mathcal{N}=4$ superYang-Mills is a vector, four chiral fermions, and six real bosons, all in the adjoint of the gauge group:

$$
\begin{aligned}
& A_{\mu} \\
& \lambda_{1} \lambda_{2} \lambda_{3} \lambda_{4} \\
& X_{1} X_{2} X_{3} X_{4} X_{5} X_{6} \text {. }
\end{aligned}
$$

These can be grouped into $\mathcal{N}=1$ multiplets in various equivalent ways. The one we will have in mind is $\left(A_{\mu}, \lambda_{4}\right)$ for the $\mathcal{N}=1$ vector multiplet and $\left(\lambda_{1}, X_{1}, X_{2}\right),\left(\lambda_{2}, X_{3}, X_{4}\right)$, and $\left(\lambda_{3}, X_{5}, X_{6}\right)$ for the adjoint chiral multiplets-also denoted by chiral superfields $\Phi_{1}, \Phi_{2}, \Phi_{3}$, whose complex scalar components are $\phi_{1}, \phi_{2}, \phi_{3}$. The superpotential of the undeformed $\mathcal{N}=4$ theory is

$$
W=\operatorname{tr} \Phi_{1}\left[\Phi_{2}, \Phi_{3}\right] .
$$

Supergravity scalars will typically be denoted $\varphi_{\Delta}$, where $\Delta$ indicates the dimension of the corresponding gauge singlet operator $\mathcal{O}_{\Delta}$. Units

\footnotetext{
${ }^{4}$ This interpretation was suggested to me by J. Polchinski and J. Maldacena.
} 
will be chosen such that $L$, the radius of curvature in the asymptotically $A d S_{5}$ region, is 1 .

\subsection{One massive adjoint chiral}

Our first example is the category of flows that arise from giving a mass to a single adjoint chiral superfield in the $\mathcal{N}=4$ langrangian [18]. The mass deformation is specified by profiles for two scalars, $\varphi_{2}$ and $\varphi_{3}$, dual to a boson mass term $\mathcal{O}_{2}$ of dimension 2 and a fermion mass term $\mathcal{O}_{3}$ of dimension 3. Explicitly,

$$
\begin{aligned}
& \mathcal{O}_{2}=\operatorname{tr}\left(-X_{1}^{2}-X_{2}^{2}-X_{3}^{2}-X_{4}^{2}+2 X_{5}^{2}+2 X_{6}^{2}\right) \\
& \mathcal{O}_{3}=\operatorname{tr}\left(\lambda_{4} \lambda_{4}+\phi_{1}\left[\phi_{2}, \phi_{3}\right]\right)+\text { h.c. }
\end{aligned}
$$

The operator $\mathcal{O}_{2}$ in (37) looks like the wrong mass deformation: adding a positive multiple of it to the lagrangian will result in negative mass squared for four of the six real scalars. What we really aim to do is add $\int d^{2} \theta \frac{1}{2} m \operatorname{tr} \Phi_{3}^{2}+h . c$. to the lagrangian. The operator $\mathcal{O}_{3}$ in (37) is precisely the dimension 3 term in this deformation. But the dimension 2 term is just $\operatorname{tr}\left(X_{5}^{2}+X_{6}^{2}\right)$. What saves the day is that $\varphi_{3}^{2}$ can couple to the $S O(6)$ invariant combination $\operatorname{tr} \sum_{I=1}^{6} X_{I}^{2}$. In a supersymmetric background involving the $e^{-r}$ part of $\varphi_{3}$ and the $r e^{-2 r}$ part of $\varphi_{2}$, the $\varphi_{3}^{2}$ coupling to the $S O(6)$ singlet mass term must precisely cancel out the negative terms in the $\mathcal{O}_{2}$ of (37), leaving only the desired $\operatorname{tr}\left(X_{5}^{2}+X_{6}^{2}\right)$. Similar quadratic couplings will be important in sections 4.2 and 4.3 .

The scalars $\varphi_{2}$ and $\varphi_{3}$ are canonically normalized, and the potential and superpotential read [18]

$$
\begin{aligned}
V\left(\varphi_{2}, \varphi_{3}\right) & =\left[-\rho^{2}+\frac{\cosh 2 \varphi_{3}-3}{4 \rho^{4}}+\frac{\rho^{8}}{8}\left(\cosh 2 \varphi_{3}-1\right)\right]\left(\cosh 2 \varphi_{3}+1\right) \\
W\left(\varphi_{2}, \varphi_{3}\right) & =\frac{1}{2} \cosh 2 \varphi_{3}\left(\rho^{4}-\frac{2}{\rho^{2}}\right)-\left(\frac{3}{2} \rho^{4}+\frac{1}{\rho^{2}}\right)
\end{aligned}
$$

where we have defined $\rho=e^{\varphi_{2} / \sqrt{6}}$. It is consistent to set all other scalars to 0 : locally $V$ is stationary perpendicular to the $\varphi_{2}-\varphi_{3}$ plane. There is a one-parameter family of flows emanating from the origin, which is the maximally symmetric point representing unperturbed $\mathcal{N}=4$ gauge theory. The trajectories have only been found numerically. Their 
asymptotics for large $r$ (the ultraviolet) was described in [18]:

$$
\varphi_{3} \sim a_{0} e^{-r} \quad \varphi_{2} \sim \frac{4}{\sqrt{6}} a_{0}^{2} r e^{-2 r}+a_{1} e^{-2 r} .
$$

Unsurprisingly, the leading $r e^{-2 r}$ behavior of $\varphi_{2}$ is fixed in terms of the leading $e^{-r}$ behavior of $\varphi_{3}$ : this reflects the fact that the boson mass term and the fermion mass term are related by supersymmetry. One can parametrize the gradient flow trajectories with the quantity

$$
\tilde{a}_{1}=\frac{a_{1}}{a_{0}^{2}}+\frac{4}{\sqrt{6}} \log a_{0},
$$

which is invariant under additive shifts of $r$. It is a dimensionless measure of $\left\langle\mathcal{O}_{2}\right\rangle$ in units set by the scale of the mass deformation. $W$ and $V$ are symmetric under $\varphi_{3} \rightarrow-\varphi_{3}$, and from now on we will simplify our discussion by considering only flows with $\varphi_{3} \geq 0$.

Curiously; it was found in [18] that $\tilde{a}_{1}=\tilde{a}_{1}^{(c)}=-1.4694 \ldots$ corresponds to the conformal vacuum of the theory, where $\left\langle\mathcal{O}_{2}\right\rangle=0$ and the supergravity flow terminates at the critical point found in [16]. By comparison with flows describing states on the Coulomb branch of $\mathcal{N}=4$ gauge theory, one can establish that $\tilde{a}_{1}>\tilde{a}_{1}^{(c)}$ corresponds to negative $\left\langle\mathcal{O}_{2}\right\rangle$. Positive $\left\langle\mathcal{O}_{2}\right\rangle$ should be impossible because we have made $X_{5}$ and $X_{6}$ massive. To put it another way, the infrared fixed point theory has a Coulomb branch parametrized by color singlet combinations of $X_{1}$ through $X_{4}$. The states with negative $\left\langle\mathcal{O}_{2}\right\rangle$ are $S U(2)_{\text {global }} \times U(1)_{R^{-}}$ symmetric states on that Coulomb branch. States with positive $\left\langle\mathcal{O}_{2}\right\rangle$ are unphysical. In figure $4(\mathrm{~b})$, the $\left\langle\mathcal{O}_{2}\right\rangle<0$ trajectories are the ones to the right of the critical trajectory which ends up at the one-quarter supersymmetric fixed point. The ones to the left are supposed to be ruled out.

Felicitously, the condition (2) accepts trajectories with $\tilde{a}_{1} \geq \tilde{a}_{1}^{(c)}$ but rules out those with $\tilde{a}_{1}<\tilde{a}_{1}^{(c)}$. The horizontal line along the $\varphi_{2}$ axis in the solution set $\mathcal{P}$ (defined in (28)) represents Coulomb branch flows of the $\mathcal{N}=4$ gauge theory. When these flows are lifted back to ten dimensions, the $\varphi_{2}>0$ direction corresponds to an $S^{3}$ shell of D3-branes in the hyperplane spanned by $x_{1}$ through $x_{4}$, while the $\varphi_{2}<0$ direction corresponds to a uniform disk of D3-branes in the plane spanned by $x_{5}$ and $x_{6}$. Because the contours of $W$ form a trough around the $\varphi_{2}$ axis for large positive $\varphi_{2}$, the $\tilde{a}_{1}>\tilde{a}_{1}^{(c)}$ trajectories are 


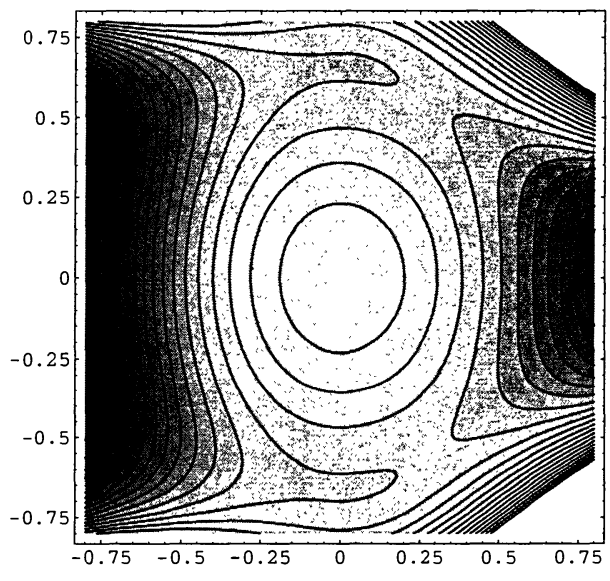

(a)

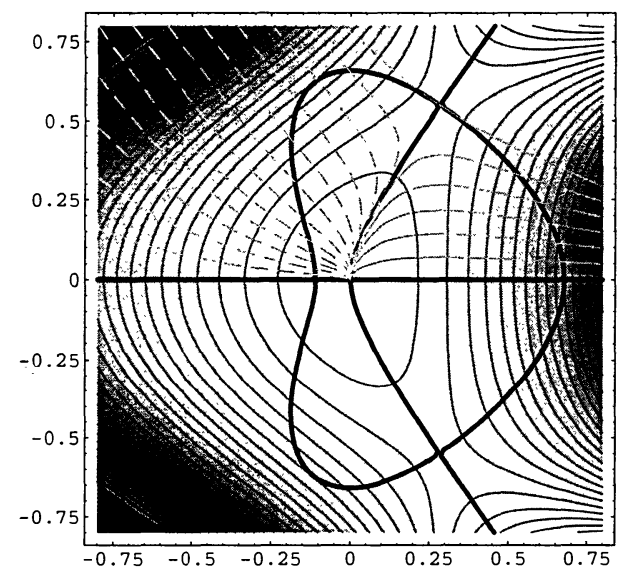

(b)

Figure 4: (a) Contours of $V\left(\varphi_{2}, \varphi_{3}\right)$, with $\varphi_{2}$ on the horizontal axis and $\varphi_{3}$ on the vertical axis. (b) Contours of $W\left(\varphi_{2}, \varphi_{3}\right)$, with the solution set $\mathcal{P}$ and some typical gradient flow trajectories superimposed. In both contour plots, lighter regions correspond to higher elevations. The undashed trajectories proceeding asymptotically due east are Coulomb branch states, and correspond to entry (B) in figure 3. The trajectory which ends at the saddle point represents the infrared conformal fixed point, and corresponds to entry $(\mathrm{C})$ in figure 3 . The dashed trajectories violate (2). 
drawn to this axis. The very low energy Coulomb branch physics of these flows does not depend on whether the adjoints $X_{5}$ and $X_{6}$ are given a mass. In contrast to this case, the contours of $W$ form a ridge around the negative $\varphi_{2}$ axis, and the $\tilde{a}_{1}<\tilde{a}_{1}^{(c)}$ trajectories are repelled from this axis. These trajectories are asymptotically parallel to one another, and they are ruled out by (2) because $V(\vec{\varphi}(r)) \rightarrow+\infty$ as $A(r) \rightarrow-\infty$. The flow exactly along the negative $\varphi_{2}$ axis represents sensible $\mathcal{N}=4$ Coulomb branch physics in the $X_{5}$ and $X_{6}$ directions; but this part of the moduli space is lifted completely when $X_{5}$ and $X_{6}$ are given a mass, and the trajectories are appropriately defocused as a result.

It turns out, as claimed after (28), that all the trajectories permitted by (2) run asymptotically parallel to some curve in $\mathcal{P}$. In fact, all trajectories converge to some curve in $\mathcal{P}$ in the far infrared. The segment of $\mathcal{P}$ stretching from the origin to the one-quarter supersymmetric saddle point is an excellent approximation to a gradient flow trajectory. The part of the curve in $\mathcal{P}$ which extends northeast from the one-quarter supersymmetric point does not describe a deformation of $\mathcal{N}=4$ super-Yang-Mills.

The flows along the positive and negative $\varphi_{2}$ axes were first considered in ten dimensions [6] as zero-temperature, zero-spin limits of near-extremal D3-brane solutions with some angular momenta turned on. Specifically, the positive $\varphi_{2}$ trajectory comes from solutions with equal $J_{12}$ and $J_{34}$ turned on, while the negative $\varphi_{2}$ trajectory comes from solutions with $J_{56}$. In five dimensions these angular momenta are interpreted as charges under the $U(1)^{3}$ Cartan subalgebra of the $S O(6)$ gauge group, and the spinning D3-branes are represented as charged black holes [41, 42]. The time components of the gauge fields in fivedimensional supergravity act as "voltages," or "chemical potentials," for conserved $R$-currents in the gauge theory. The positive and negative $\varphi_{2}$ flows are examples of states which can be approached by tuning these chemical potentials to zero in fixed ratio with an appropriate power of the temperature. There is a thermodynamic instability that sets in at some point in this limiting process [43], but that is somewhat outside our current scope of interest. 


\subsection{Two massive adjoint chirals}

Our next example is $\mathcal{N}=4$ super-Yang-Mills broken to $\mathcal{N}=2$ by a mass term for an adjoint hypermultiplet. The analysis is based on joint work with $\mathrm{K}$. Pilch and $\mathrm{N}$. Warner [40]. In $\mathcal{N}=1$ language, we are giving equal but opposite masses to two adjoint chiral multiplets, which we take to be $\Phi_{1}$ and $\Phi_{2}$-exactly the chiral adjoints which we left massless in the previous example. The field theory was studied in [44]. The supergravity [40] involves the same scalar $\varphi_{2}$ in the $\mathbf{2 0}^{\prime}$ of $S O(6)$ as we had in the previous example, but a different scalar $\tilde{\varphi}_{3}$ in the $\mathbf{1 0}+\overline{\mathbf{1 0}}$. The dual operators are

$$
\begin{aligned}
& \mathcal{O}_{2}=\operatorname{tr}\left(-X_{1}^{2}-X_{2}^{2}-X_{3}^{2}-X_{4}^{2}+2 X_{5}^{2}+2 X_{6}^{2}\right) \\
& \tilde{\mathcal{O}}_{3}=\operatorname{tr}\left(\lambda_{1} \lambda_{1}-\lambda_{2} \lambda_{2}+(\text { scalar trilinear })\right)+\text { h.c. }
\end{aligned}
$$

As in the previous example, the square of $\tilde{\varphi}_{3}$ can couple to the $S O(6)$ singlet mass operator. The combination of this operator and $-\mathcal{O}_{2}$ that supersymmetry demands is proportional to $\operatorname{tr}\left(X_{1}^{2}+X_{2}^{2}+X_{3}^{2}+X_{4}^{2}\right)$. Both $\varphi_{2}$ and $\tilde{\varphi}_{3}$ are canonically normalized, and the potential and superpotential read

$$
\begin{aligned}
V\left(\varphi_{2}, \tilde{\varphi}_{3}\right) & =\frac{1}{4} e^{-\frac{4}{\sqrt{6}} \varphi_{2}}\left(-4-8 e^{\sqrt{6} \varphi_{2}} \cosh \sqrt{2} \tilde{\varphi}_{3}+e^{2 \sqrt{6} \varphi_{2}} \sinh ^{2} \sqrt{2} \tilde{\varphi}_{3}\right) \\
W\left(\varphi_{2}, \tilde{\varphi}_{3}\right) & =-2 e^{-\frac{2}{\sqrt{6}} \varphi_{2}}-e^{\frac{4}{\sqrt{6}} \varphi_{2}} \cosh \sqrt{2} \tilde{\varphi}_{3} .
\end{aligned}
$$

The asymptotics at large $r$ is

$$
\tilde{\varphi}_{3} \sim b_{0} e^{-r} \quad \varphi_{2} \sim-\frac{2}{\sqrt{6}} b_{0}^{2} r e^{-r}+b_{1} e^{-2 r},
$$

and the invariant which parametrizes the one-parameter family of flows emanating from the origin is

$$
\tilde{b}_{1}=\frac{b_{1}}{b_{0}^{2}}-\frac{2}{\sqrt{6}} \log b_{0} .
$$

In figure 5 we exhibit contours of $V$ and $W$, the solution set $\mathcal{P}$, and some characteristic gradient flow trajectories (obtained numerically). Because of the $\tilde{\varphi}_{3} \rightarrow-\tilde{\varphi}_{3}$ symmetry, we can restrict our attention to flows with $\tilde{\varphi}_{3} \geq 0$. When $\tilde{b}_{1}=\tilde{b}_{1}^{(c)} \approx 0.125 \ldots{ }^{5}$ the flow is asymptotic to the curve in $\mathcal{P}$ which follows the obvious northwest ridgeline

\footnotetext{
${ }^{5}$ Random jitter near the maximally supersymmetric point makes this number (and also $\tilde{a}_{1}^{(c)}$ ) surprisingly hard to pin down numerically.
} 
of $W$ (see figure 5). The condition (2) allows this trajectory and all others to the left of it: $\tilde{b}_{1} \leq \tilde{b}_{1}^{(c)}$. The trajectories with $\tilde{b}_{1}<\tilde{b}_{1}^{(c)}$ run asymptotically parallel to the horizontal axis: this is an explicit example where (27) is satisfied without the trajectories actually approaching any curve in $\mathcal{P}$. (States on the Coulomb branch which fail to preserve an $S O(n) \times S O(6-n)$ global symmetry are another example). The $\tilde{b}_{1}=\tilde{b}_{1}^{(c)}$ trajectory is well approximated by a curve in $\mathcal{P}$. The trajectories with $\tilde{b}_{1}>\tilde{b}_{1}^{(c)}$ proceed down the northeast face of $W$. As is evident from comparing figure $5(\mathrm{a})$ with figure $5(\mathrm{~b})$, this is a case where $(27)$ is violated. No gradient flow trajectory of $W$ ends up at the saddle points near the northeast and southeast corners of figure $3(\mathrm{a})$ : these points correspond to $A d S_{5}$ vacua which break all supersymmetry (see Table 1, entry (iv) of [16]).

There is a natural field theory interpretation of these flows, inspired by [37], though it must be regarded as speculative in the absence of more detailed tests. The far infrared theory is large- $N$ Seiberg-Witten theory, and the Seiberg-Witten curve [45] is [46, 47]

$$
y^{2}=\prod_{k=1}^{N}\left(x-\xi_{k}\right)^{2}-\Lambda^{2 N},
$$

where $\Lambda$ is the dynamically generated scale and $\xi_{k}$ are parameters determining the position on the moduli space. In [37] it was suggested that the enhançon singularity corresponds to Seiberg-Witten theory at the "origin" of moduli space $\xi_{k}=0$, and that the branch points $x_{k}=\Lambda e^{i \pi k / N}$ correspond to the positions of branes in a ring around the enhançon. The $\tilde{b}_{1}=\tilde{b}_{1}^{(c)}$ flow seems most likely to be dual to this same point in moduli space, where now the complex variable $x$ parametrizes the directions of the adjoints $X_{5}$ and $X_{6}$ which remain massless. This interpretation is supported by the fact that the supergravity geometry preserves a $U(1)$ symmetry which rotates $X_{5}$ and $X_{6}$. Because the $\tilde{b}_{1}>\tilde{b}_{1}^{(c)}$ flows "interpolate" between the ridgeline flow and the negative $\varphi_{2}$ axis, and because the negative $\varphi_{2}$ axis is known to correspond to a state on the Coulomb branch of the undeformed $\mathcal{N}=4$ theory where the D3-branes are arranged in a uniform disk in the $X_{5}-X_{6}$ direction, the most obvious candidate for a field theory dual of the $\tilde{b}_{1}>\tilde{b}_{1}^{(c)}$ flows is a point on moduli space where the $\xi_{i}$ are uniformly distributed on a disk. When the radius of that disk is much larger than $\Lambda$, the lowenergy physics approaches the Coulomb branch physics of $\mathcal{N}=4$ gauge 


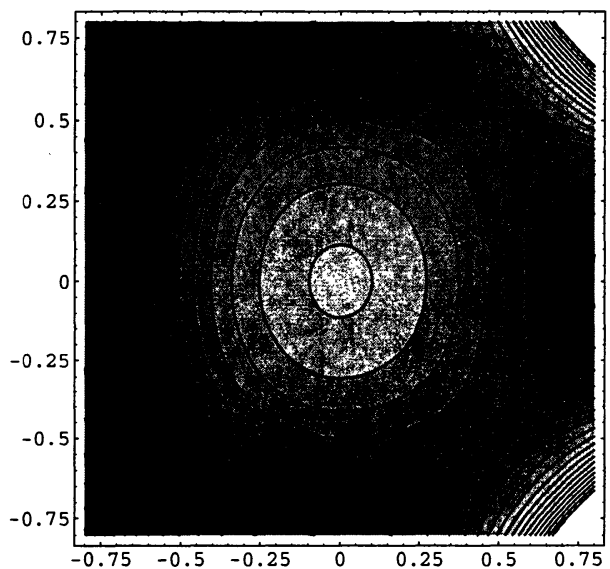

(a)

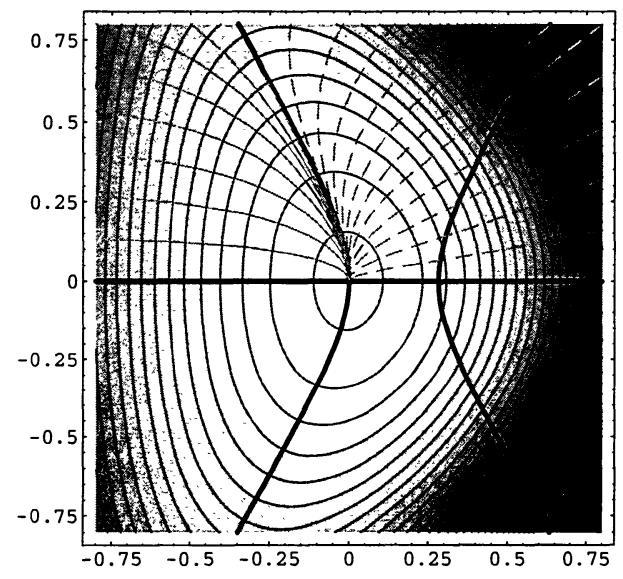

(b)

Figure 5: (a) Contours of $V\left(\varphi_{2}, \tilde{\varphi}_{3}\right)$, with $\varphi_{2}$ on the horizontal axis and $\tilde{\varphi}_{3}$ on the vertical axis. (b) Contours of $W\left(\varphi_{2}, \varphi_{3}\right)$, with the solution set $\mathcal{P}$ and some typical gradient flow trajectories superimposed. In both contour plots, lighter regions correspond to higher elevations. The solid trajectories which proceed asymptotically due west are states on the Coulomb branch of the $\mathcal{N}=2$ gauge theory, and correspond to entry (D) in figure 3. The unique solid trajectory which proceeds northwest nearly along a curve in $\mathcal{P}$ is the best candidate for a vacuum which can support finite temperature. The dashed trajectories violate (2). 
theory.

On this interpretation, the trajectories with $\tilde{b}_{1}<\tilde{b}_{1}^{(c)}$ are unphysical. Without the mass deformation, the disk of D3-branes in the $X_{5}-X_{6}$ directions can shrink to a point and then re-expand as an $S^{3}$ shell in the $X_{1}$ through $X_{4}$ directions. This is exactly the picture found in [8]. But when we make $X_{1}$ through $X_{4}$ massive, this part of the moduli space disappears, and we are in the position of attempting to give a negative VEV to $\operatorname{tr}\left(X_{5}^{2}+X_{6}^{2}\right)$. All this fits in perfectly with (2) which indeed rules out the $\tilde{b}_{1}<\tilde{b}_{1}^{(c)}$ trajectories. To lend further support to the picture we have presented, it would nice if one could show that finite temperature in the field theory draws the vacuum state to the point where all $\xi_{k}=0$. Correspondingly in the supergravity, one would hope that a horizon with small but finite temperature draws the scalars onto the $\tilde{b}_{1}=\tilde{b}_{1}^{(c)}$ trajectory.

\subsection{Three massive adjoint chirals}

Our last example is the category of flows believed to be dual to $\mathcal{N}=4$ super-Yang-Mills deformed by a uniform mass for all three chiral adjoint superfields [20]: $W \rightarrow W+\frac{m}{2} \operatorname{tr} \sum_{i} \Phi_{i}^{2}$. Naively, the infrared theory is $\mathcal{N}=1$ super-QCD. In fact, the model has many vacua, corresponding to non-commutative VEV's for the massive scalars $\phi_{i}$ which form a representation of $S U(2)[48,44]$.

The deformation of the lagrangian consists of a dimension 3 operator, which preserves $S O(3)$ and gives a uniform mass to three adjoint fermions; and a dimension 2 operator, which is just the $S O(6)$ symmetric mass term for all the scalars. The supergravity scalar dual to the dimension 3 mass term will be denoted $\varphi_{m}$. We will also be interested in the dimension three operator $\mathcal{O}_{3}$ which includes the $\mathcal{N}=1$ gaugino bilinear. The dual scalar will be denoted $\varphi_{3}$. This is the same scalar which was used in section 4.1 to introduce a mass for one species of fermion. Both $\varphi_{m}$ and $\varphi_{3}$ are canonically normalized. The potential 
and the superpotential are

$$
\begin{aligned}
V\left(\varphi_{m}, \varphi_{3}\right) & =-\frac{3}{8}\left(\cosh ^{2} \frac{2 \varphi_{m}}{\sqrt{3}}+4 \cosh \frac{2 \varphi_{m}}{\sqrt{3}} \cosh 2 \varphi_{3}-\cosh ^{2} 2 \varphi_{3}+4\right) \\
W\left(\varphi_{m}, \varphi_{3}\right) & =-\frac{3}{2}\left(\cosh \frac{2 \varphi_{m}}{\sqrt{3}}+\cosh 2 \varphi_{3}\right) .
\end{aligned}
$$

A striking feature of this case as compared to the previous two is that there is only one scalar that takes care of the mass deformation. In fact this is entirely appropriate: there is no supergravity scalar which couples linearly to the $S O(6)$-invariant boson mass term, $\operatorname{tr} \sum_{I} X_{I}^{2}$. The scalar $\varphi_{m}$ couples linearly to the $S O(3)$-invariant fermion mass term, and it can couple quadratically to the $\operatorname{tr} \sum_{I} X_{I}^{2}$. Demanding supersymmetry guarantees that the boson and fermion mass terms will come in the right ratio.

There is a one-parameter family of gradient flow trajectories for the superpotential in (46), all of which can be displayed in analytic form [20]:

$$
\begin{aligned}
A & =\frac{1}{2} \log \left[2 \sinh \left(r-C_{1}\right)\right]+\frac{1}{6} \log \left[2 \sinh \left(3 r-C_{2}\right)\right] \\
\varphi_{m}(r) & =\frac{\sqrt{3}}{2} \log \frac{1+e^{-r+C_{1}}}{1-e^{-r+C_{1}}} \\
\varphi_{3}(r) & =\frac{1}{2} \log \frac{1+e^{-3 r+C_{2}}}{1-e^{-3 r+C_{2}}} .
\end{aligned}
$$

The behavior $\varphi_{m}(r) \sim e^{-r}$ for large $r$ indicates that there is a relevant perturbation of the lagrangian. The parameter $C_{1}$ amounts to setting the scale of this mass deformation. On the other hand, $\varphi_{3}(r) \sim e^{-3 r}$ signals a VEV for the operator $\mathcal{O}_{3}$. The invariant quantity which parametrizes the trajectories is $\lim _{r \rightarrow \infty} \varphi_{3}(r) / \varphi_{m}(r)^{3}$. It is related to the VEV of $\mathcal{O}_{3}$ :

$$
\lim _{r \rightarrow \infty} \frac{\varphi_{3}(r)}{\varphi_{m}(r)^{3}}=\frac{1}{\sqrt{27}} e^{C_{2}-3 C_{1}}=\frac{c_{1}}{N^{2}} \frac{\left\langle\mathcal{O}_{3}\right\rangle}{m^{3}}
$$

where $\Lambda$ is the dynamical mass scale and $c_{1}$ is a constant of order unity which can be determined once the normalization of $\mathcal{O}_{3}$ and $\Lambda$ are unambiguously specified. To understand the factor of $1 / N^{2}$ in (48), recall that there is an overall factor of $1 / G_{5}$ in front of the classical 
supergravity action. When this action is used to compute a correlator of boundary operators, three powers of the radius of $A d S_{5}$ combine with $1 / G_{5}$ to give an overall factor of $N^{2}$. An example is the stress tensor two-point function: suppressing Lorentz indices, the correlation function that one computes in the classical supergravity approximation is $\langle T(x) T(0)\rangle \sim c / x^{8}$ with $c \sim N^{2}$. More generally, the operators to which canonically normalized supergravity scalars couple with strength one (that is, without explicit factors of $N$ in the coupling) have 2-point functions which scale as $N^{2}$. An order 1 profile for a supergravity scalar is dual to either an order 1 coefficient for the dual operator added to the lagrangian, or an order $N^{2}$ VEV for that operator. In particular, when $\varphi_{3}$ and $\varphi_{m}$ of order $1,\left\langle\mathcal{O}_{3}\right\rangle$ is of order $N^{2}$. Thus for (48) to be consistent with $c_{1}$ of order 1 it is necessary to include the explicit $1 / N^{2}$.

On the supergravity side, we can try to use (2) to rule out some trajectories. Contour plots of $V$ and $W$, the solution set $\mathcal{P}$, and some typical gradient flow trajectories are shown in figure 6 . We can take advantage of the $\varphi_{m} \rightarrow-\varphi_{m}$ and $\varphi_{3} \rightarrow-\varphi_{3}$ symmetries to restrict our attention to flows in the northeast corner of the plots. The condition (2) allows flows with $C_{2} \leq 3 C_{1}$ : the others violate the steepness condition $\zeta \leq \sqrt{8 / 3}$. The curves in $\mathcal{P}$ give good approximations to gradient flow trajectories in the infrared, but the approximation breaks down more badly than in the previous examples as one proceeds toward the ultraviolet. The saddle points on the vertical axis of (a) are the unstable $S U(3)$ critical points.

The field theory is more complicated than in the previous examples, and it is correspondingly more difficult to identify the vacua corresponding to different trajectories in figure 6(b). After the mass deformation, the superpotential in the field theory is

$$
W=\operatorname{tr}\left(\frac{m}{2} \sum_{i=1}^{3} \Phi_{i}^{2}+\Phi_{1}\left[\Phi_{2}, \Phi_{3}\right]\right),
$$

and the F-flatness conditions read

$$
\frac{\partial W}{\partial \phi_{k}}=m \phi_{k}+\left[\phi_{k+1}, \phi_{k+2}\right]=0
$$

where we identify the index $i$ on $\phi_{i}$ modulo 3 . Thus the $\phi_{i}$ should form some $N$-dimensional representation of $S U(2)$ (not necessarily irreducible). Imposing the D-flatness conditions and identifying gaugeequivalent configurations can be achieved simultaneously by modding 


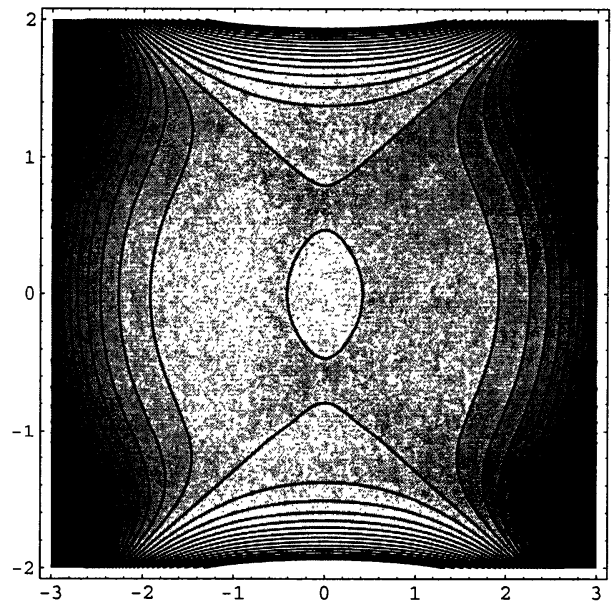

(a)

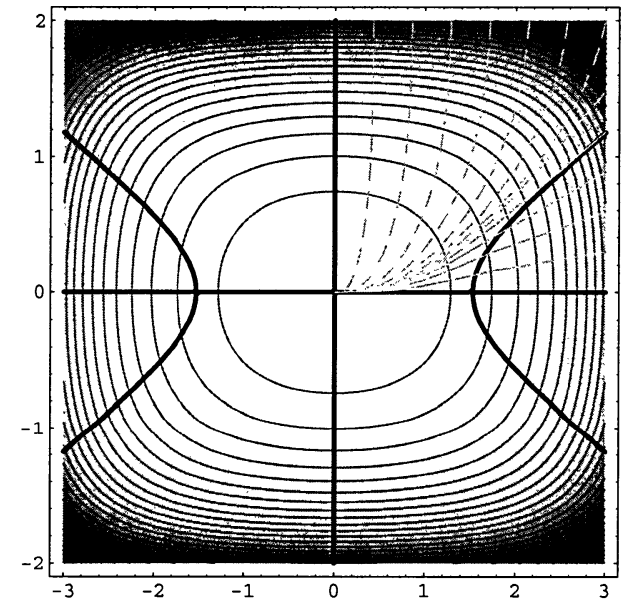

(b)

Figure 6: (a) Contours of $V\left(\varphi_{m}, \varphi_{3}\right)$, with $\varphi_{m}$ on the horizontal axis and $\varphi_{3}$ on the vertical axis. (b) Contours of $W\left(\varphi_{m}, \varphi_{3}\right)$, with the solution set $\mathcal{P}$ and some typical gradient flow trajectories superimposed. In both contour plots, lighter regions correspond to higher elevations. Note that the aspect ratio is not unity, so gradient flow trajectories don't appear to be contour-orthogonal. The undashed trajectory which proceeds northeast and is asymptotic to a curve in $\mathcal{P}$ is the clearest candidate for a massive vacuum of the theory. It corresponds to entry (G) in figure 3. The other undashed trajectories could also be well defined vacua, and correspond to entry $(F)$ in figure 3. 
out by the action of the complexified gauge group. Roughly speaking, one can think of the representations of $S U(2)$ as non-commutative spheres, whose radii are the quadratic Casimirs of the representations. The radius is largest for the $N$-dimensional irreducible representation. ${ }^{6}$ In this case,

$$
\left\langle\operatorname{tr} \phi_{1}\left[\phi_{2}, \phi_{3}\right]\right\rangle \approx \frac{N^{3}}{12} m^{3} .
$$

Going to the opposite extreme, one could satisfy (50) trivially by setting all the $\phi_{i}$ to 0 . In this case, the gauge group is completely unbroken, and there is a gaugino condensate, which according to the standard analysis is

$$
\left\langle\operatorname{tr} \lambda_{4} \lambda_{4}\right\rangle \approx c_{2} e^{2 \pi i k / N} N \Lambda^{3},
$$

where $c_{2}$ is another constant of order unity, $\Lambda$ is the scale of the low-energy $\mathcal{N}=1$ theory, and $k$ can run from 1 to $N$ (see for example $[50,51,52,53,54,55])$. Again up to factors of order unity, $\Lambda=m \exp \left(-\frac{8 \pi^{2}}{3 g^{2} N}\right)$ where $g$ is the gauge coupling at the $\mathcal{N}=4$ ultraviolet fixed point. The overall factor of $N$ in (52) ensures that domain walls between adjacent vacua have tension scaling as $N$. To see this recall that the exact superpotential has one more power of $N$ than the gaugino condensate; but domain wall tensions are given by the change of $W$ across the domain wall, and $W$ changes by a phase $e^{2 \pi i / N}$ from one vacuum to the next [56].

Naively, the operator $\mathcal{O}_{3}$ is $\operatorname{tr}\left(\lambda_{4} \lambda_{4}+\phi_{1}\left[\phi_{2}, \phi_{3}\right]\right)+$ h.c., as in (37). Both the fermion bilinear and the scalar trilinear are manifestly $S U(3)$ invariant. The VEV of the first term in the trivial vacuum is order $N$, while the VEV of the second is order $N^{3}$ in the irreducible vacuum. Classical supergravity effects correspond to VEV's of order $N^{2}$, so the first behavior appears unobservably small, while the second seems un-

\footnotetext{
${ }^{6}$ Similar non-commutative configurations were studied in the test-brane approximation in [49]. In fact, one can see a hint of the physics of (50) by putting D3-branes in a constant background field $G_{I J K}$, where $G_{(3)}$ is the complex three-form field strength of type IIB supergravity, and $I J K$ are indices in the directions perpendicular to the D3-branes. $G_{(3)}$ has to be involved in the ten-dimensional version of the solutions specified in (47) (indeed in the ten-dimensional version of all the solutions presented in this section with the exception of the Coulomb branch trajectories of the undeformed $\mathcal{N}=4$ theory). If $*_{6} G_{(3)}=i G_{(3)}$, where $*_{6}$ denotes the Hodge dual in the $I J K$ directions, then in the approach of [49] there remain commutative flat directions for the D3-branes to spread out in. Possibly an improved treatment with non-constant $G_{(3)}$ could approximate the physics of (50) more closely.
} 
observably large! We suggest however that

$$
\mathcal{O}_{3}=\operatorname{tr}\left(\lambda_{4} \lambda_{4}+\sum_{i=1}^{3} \phi_{i} \frac{\partial W}{\partial \phi_{i}}\right)+\text { h.c. }
$$

where $W$ is the deformed superpotential, (49), and we are not attempting to be precise about factors of order unity on either the fermion or scalar terms. Classically, the scalar terms have vanishing VEV in any vacuum. However, the Konishi relation gives

$$
\sum_{i=1}^{3}\left\langle\operatorname{tr} \phi_{i} \frac{\partial W}{\partial \phi_{i}}\right\rangle=N\left\langle\operatorname{tr} \lambda_{4} \lambda_{4}\right\rangle .
$$

Thus if $\left\langle\operatorname{tr} \lambda_{4} \lambda_{4}\right\rangle$ is order $N$, as the standard field theory analysis indicates, then $\left\langle\mathcal{O}_{3}\right\rangle$ is order $N^{2}$.

Because the standard field theory analysis of the trivial confining vacua indicates a $\mathrm{VEV}$ for $\mathcal{O}_{3}$ which is of the right order to be observed as a classical effect in supergravity, the natural guess is that these vacua (or one of them, say the $k=0$ vacuum) should be identified as the $C_{2}=3 C_{1}$ trajectory in figure $6(\mathrm{~b})$. This trajectory is distinguished in that it is the last one permitted by the condition (2). It has a smaller value of $\zeta$ than all the other trajectories, and it is asymptotic to a curve in $\mathcal{P}$, which makes it the most plausible candidate for a flow with near-extremal generalizations which do not involve deforming the lagrangian.

The other trajectories permitted by (2) could have one of several interpretations, and without further investigation we cannot judge which interpretation is correct. First, they might be in some way unstable, and so correspond to no well-defined field theory vacuum. Second, they might be massless vacua corresponding to embeddings of $S U(2)$ in $S U(N)$ which leave factors of $U(1)$ unbroken. Third, they might be massive vacua where $S U(N)$ is broken down to some $S U(N / p)$ subgroup by a representation of $S U(2)$ which can be written as $N / p$ blocks of dimension $p \times p$. A recent analysis [57] indicates yet one more possibility. The claim of [57] is that the standard analysis (52) of the gaugino condensate is dramatically altered at strong coupling, and that in the large $N$, large $g_{Y M}^{2} N$ limit the regular $N$-gon of vacua for the trivial representation of $S U(2)$ degenerates to points on a line segment. These 
points are not evenly distributed; rather,

$$
\left\langle\operatorname{tr} \lambda_{4} \lambda_{4}\right\rangle=N m^{3} / j^{2} \quad \text { where } j=1,2,3, \ldots
$$

We have rescaled $m$ to conform with our conventions, set $g_{Y M}=1$, and dropped various factors of order unity. Since $\left\langle\operatorname{tr} \lambda_{4} \lambda_{4}\right\rangle$ still scales as $N$, the previous arguments that $\left\langle\mathcal{O}_{3}\right\rangle$ should be observable as a classical effect in supergravity still hold, and we might still expect the $C_{2}=3 C_{1}$ trajectory to correspond to the $j=1$ vacuum (which is the large $\lambda$ limit of the $k=0$ vacuum in the standard analysis (52)). Then some of the $C_{2}<3 C_{1}$ trajectories might be $j>1$ vacua.

The trajectories with $C_{2}>3 C_{1}$ violate (2) and run asymptotically parallel to the $\varphi_{3}$-only trajectory. This trajectory seems to be a particularly clear case of an unphysical geometry: the lagrangian is undeformed, a gaugino condensate has no reason to form, the flat directions are $\left[X_{I}, X_{J}\right]=0$, and yet we are trying to give a nonzero VEV to $\mathcal{O}_{3}=\operatorname{tr}\left(\lambda_{4} \lambda_{4}+\phi_{1}\left[\phi_{2}, \phi_{3}\right]\right)$. By extension it is plausible to rule out all the $C_{2}>3 C_{1}$ trajectories, since they have the same behavior in the infrared. Incidentally, the "universal solution" of Horava-Witten theory compactified on a Calabi-Yau manifold [58] develops the same singularity as the $C_{2}>3 C_{1}$ flows if the negative tension "hidden sector" end-of-the-world brane is taken to infinite redshift. The low-energy limit of the hidden sector gauge theory is pure $\mathcal{N}=1$ super-YangMills theory. The unpleasant singularity could be due to neglect of the gaugino condensate in the derivation of this solution.

To sum up, what tells in favor of the condition (2) in this example is that the field theory analysis indicates VEV's for $\left\langle\mathcal{O}_{3}\right\rangle$ which are of order $N^{2}$ and bounded in magnitude. This qualitative feature is reproduced by (2) because it implies $C_{2} \leq 3 C_{1}$. It would be interesting to study the symmetries of the problem more carefully. In particular, is there a $U(1)$ symmetry in the supergravity, broken by the choice of $\varphi_{3}$, which arises from the large $N$ limit of the discrete $\mathbf{Z}_{2 N}$ symmetry? According to [57] this symmetry only pertains in the small $g_{Y M}^{2} N$ limit. 


\section{Fluctuations}

Obvious cases where the criterion (2) fails to guarantee a physical interpretation on the field theory side are flows to critical points of $V$ which violate the Breitenlohner-Freedman bound [34,35]. Two wellknown examples are flows to the $S O(5)$ symmetric critical point of $d=5 \mathcal{N}=8$ gauged supergravity, and flows to the $S U(3)$ critical point $[14,15,59,60]$. We would like to have a generalization of the Breitenlohner-Freedman bound which applies to cases which are only asymptotically $A d S_{5}$ in the ultraviolet. The obvious one is to demand that the spectral decomposition of the Lorentzian two-point function of an arbitrary color singlet operator should involve only timelike momenta. This statement can be phrased in the jargon of "AdS/QCD" as the absence of tachyonic glueballs. More generally we can think of it as stability of the field theory vacuum: a gauge singlet operator $\mathcal{O}(0)$, acting at the origin of position space on the vacuum of the dual gauge theory, should produce only states with timelike momenta. On Wick rotating to Euclidean signature, the requirement amounts to having two-point functions which are non-oscillatory at large distances, but instead fall off like powers or exponentials. This makes it clear that the criterion is indeed a generalization of the Breitenlohner-Freedman bound, since violations of that bound translate precisely into gauge singlet operators with complex dimension.

The restriction to timelike momenta is straightforward to implement in AdS/CFT because the spectrum of momenta-squared is identical to the spectrum of an appropriately defined one-dimensional Schrodinger hamiltonian. This identification is a direct consequence of the prescription of $[2,3]$, and it is the basis of all AdS-glueball calculations following $[61,62]$. Since it has been discussed, implicitly or explicitly, in many other places in the literature (for instance $[61,62,8,9,63]$ ) we can afford to be brief. Our aim is simply to make the discussion of [8] more systematic.

Consider a Poincaré invariant flow solution of the form (1). It is convenient to introduce a new radial variable, $z$, such that $d r=e^{A} d z$. The solution assumes the form

$$
\begin{aligned}
d s^{2} & =e^{2 A(z)}\left(-d t^{2}+d \vec{x}^{2}+d z^{2}\right) \\
\vec{\varphi} & =\vec{\varphi}(z)
\end{aligned}
$$


Linearizing the equations of motion around this solution is tricky because linear fluctuations of the graviton couple to linear fluctuations of the scalars involved in the flow. However, scalar fluctuations $\delta \vec{\varphi}\left(x^{\mu}, z\right)$ satisfying $\delta \vec{\varphi}\left(x^{\mu}, z\right) \perp \vec{\varphi}^{\prime}(z)$ nearly decouple from the graviton. That decoupling would be exact if $\vec{\varphi}^{\prime}(z)$ didn't depend on $z$, but we may assume that $\vec{\varphi}$ changes direction only slowly in the deep infrared as $A(z) \rightarrow-\infty$. Since we are mainly interested in discerning infrared properties, little is lost in the approximation that the orthogonal $\delta \vec{\varphi}\left(x^{\mu}, z\right)$ excitations decouple from the graviton. Linearizing the wave equation $\square \vec{\varphi}=\partial V / \partial \vec{\varphi}$ leads to

$$
\square \delta \vec{\varphi}=\left.\frac{\partial^{2} V}{\partial \vec{\varphi} \partial \vec{\varphi}}\right|_{\vec{\varphi}(z)} \cdot \delta \vec{\varphi} .
$$

We can solve via separation of variables: setting $\delta \vec{\varphi}=e^{-\frac{3}{2} A(z)} e^{i k_{\mu} x^{\mu}} \vec{\psi}(z)$, one obtains directly

$$
\left[-\partial_{z}^{2}+U(z)\right] \vec{\psi}=-k^{2} \vec{\psi}
$$

where

$$
U(z)=\frac{3}{2} A^{\prime \prime}(z)+\frac{9}{4} A^{\prime}(z)^{2}+\left.e^{2 A(z)} \frac{\partial^{2} V}{\partial \vec{\varphi} \partial \vec{\varphi}}\right|_{\vec{\varphi}(z)} .
$$

$U(z)$ is a matrix acting in the space of scalars orthogonal to the flow. The Schrodinger problem (58) determines the spectrum of $k^{2}$, and in our mostly plus conventions the requirement of timelike momenta is $k^{2} \leq 0$. There are two caveats to be kept in mind. First, we used the approximation of decoupling from the graviton to derive (58). Second, if the flow geometry has a curvature singularity, supergravity gives at best an approximation to the spectrum. The curvature singularity indicates an infrared problem, so in physical cases it should be possible to smooth it out-for instance by going to finite temperature and Wick rotating so that the manifold is entirely non-singular. If the smoothing is done in the far infrared, it should only affect the behavior of $U(z)$ near the radius where $A(z) \rightarrow-\infty$. One can hope that both caveats change the spectrum only in a controllably small way: for instance, they might make the infimum of the spectrum slightly negative rather than strictly zero.

Let us now check that we recover the Breitenlohner-Freedman bound for AdS geometries. Here $e^{A(z)}=L / z$, no scalars are excited, and cur- 
vatures are bounded, so we can impose the sharp inequality

$$
\left[-\partial_{z}^{2}+U(z)\right]=\left[-\partial_{z}^{2}+\frac{15 / 4+m^{2} L^{2}}{z^{2}}\right] \geq 0 .
$$

This leads to $m^{2} L^{2} \geq-4$ by a standard Bessel function analysis.

For geometries with naked singularities, the positivity of the Schrodinger operator in (58) is essentially a condition proposed in [64] almost twenty years ago, and also considered in [65]. ${ }^{7}$ With trivial adaptations appropriate to the current context, the contents of [64] can be summarized as follows. The Schrodinger operator on the left hand side of (58) is the radial part of a linearized wave operator in a static background with a naked singularity. Let us use $\mathcal{A}$ to denote the Schrodinger operator. $\mathcal{A}$ is symmetric with respect to an appropriate inner product $(\cdot, \cdot)$ for the wave-functions. If it is also positive, then there is a natural extension (the Fredholm extension) of $\mathcal{A}$ to a self-adjoint operator on the Hilbert space of functions which is the closure under the norm $(\vec{\psi}, \vec{\psi})+(\vec{\psi}, \mathcal{A} \vec{\psi})$ of smooth functions whose support excludes both the naked singularity and the boundary of $A d S_{5}$. The existence of a selfadjoint extension of $\mathcal{A}$ is a natural criterion because it means that it is possible to define a unitary evolution equation for linearized fluctuations around the static background. The crucial step is positivity of $\mathcal{A}$, and in AdS/CFT this is equivalent to the stability of the field theory vacuum. The bulk spacetime analysis looks like it could proceed for $\mathcal{A}$ bounded below (not necessarily by zero), provided one is willing to introduce a sufficiently large $(\vec{\psi}, \vec{\psi})$ component in defining the norm. But, modulo the caveats mentioned above, the natural AdS/CFT requirement is positivity.

Suppose there is a naked singularity in the geometry at $z=z_{0}$. Let $u(z)$ denote the smallest (i.e. most negative) eigenvalue of $U(z)$. Then through almost the same Bessel function analysis that one uses to translate (60) into the Breitenlohner-Freedman bound, one finds that

$$
\liminf _{z \rightarrow z_{0}}\left(z-z_{0}\right)^{2} u(z) \geq-\frac{1}{4}
$$

is a necessary condition for $\mathcal{A}$ to be bounded below. (This is almost true; to be rigorous one must exclude the possibility that $u(z)$ oscillates rapidly).

\footnotetext{
${ }^{7}$ I thank G. Horowitz for a discussion of [65] and of the basic criterion.
} 
The conditions we have discussed should generalize to supergravity fields with spin, and to other dimensions. It was only to avoid technical difficulties that we restricted our attention to scalar fluctuations perpendicular to the flow. The general problem of diagonalizing the linearized fluctuations of all supergravity fields is computationally challenging, but the final requirement is that that the two-point functions extracted from the diagonalized equations should involve only timelike momenta in their spectral decomposition.

\section{Discussion}

Applications of AdS/CFT to non-conformal theories appears at present to be a subject of particulars, with only a few general truths. The ctheorem is one such truth $[14,18]$; the possibility of replacing second order equations of motion with first order gradient flow equations is another $[18,32,33,22]$. We have attempted to find a third, namely a criterion for what sorts of singular behavior are allowed far from the boundary of AdS. Our conjectured criterion, (2), fares reasonably well when confronted by examples in the literature. With a few exceptions, it correctly distinguishes pathological from non-pathological. The exceptions are flows to critical points which violate the Breitenlohner-Freedman bound. We have suggested that the Breitenlohner-Freedman bound is a special case of a more general requirement, namely that the spectral decomposition of two-point functions in AdS/CFT should involve only timelike momenta. The methods proposed for ruling out unphysical singularities-namely, (2) and well-behaved two-point functions-are applicable independent of supersymmetry. The examples considered in section 4 all preserve some fraction of supersymmetry, but this is only in order to have a clear understanding of the dual field theory.

The flows to critical points violating the Breitenlohner-Freedman bound are pathological cases which (2) fails to rule out. In the current literature, there are no clean exceptions to (2) in the other directionthat is, clearly physical solutions which violate (2). It would be very interesting if one could find such an exception, and to ask how and whether it supports finite temperature. In view of the examples in this paper, one's first question when addressing a putative exception must be, "Is the theory in a physical vacuum state?" 
Ideally, if the AdS/CFT map were perfectly understood, we would be able to say precisely which singularities are resolved by non-trivial infrared physics and which are not. A complete understanding of this question might lead to a complicated set of constraints on the five-dimensional bulk. The condition (2) is a simple semi-empirical rule that captures some non-trivial aspects of these constraints. A particularly clear example is a VEV for the $S U(3)$ singlet operator $\mathcal{O}_{3}=\operatorname{tr}\left(\lambda_{4} \lambda_{4}+\phi_{1}\left[\phi_{2}, \phi_{3}\right]\right)$. On the field theory side, such a VEV is forbidden unless the $\mathcal{N}=4$ lagrangian is deformed. The second term of $\mathcal{O}_{3}$ can't have a VEV because the $\mathcal{N}=4$ effective potential is flat only along directions where the $\phi_{i}$ commute. The Konishi anomaly relates the VEV for the first and second terms of $\mathcal{O}_{3}$, so that the vanishing of one implies the vanishing of the other. In supergravity, one is certainly free to perturb $A d S_{5}$ by a scalar profile indicating a VEV for $\mathcal{O}_{3}$, but at the expense of violating (2). The second order bulk equations have too many solutions if arbitrary singularities are allowed; (2) is a useful tool for weeding out the good from the bad.

What precisely is bad about the field theory dual to singularities that violate (2) has to be addressed case by case. We have already indicated the problem for $\left\langle\mathcal{O}_{3}\right\rangle \neq 0$ without a mass deformation of the $\mathcal{N}=4$ lagrangian. In sections 4.1 and 4.2 , the problem with trajectories violating (2) was that positive definite operators have negative VEV's. In section 3.2 , (2) ruled out multi-center distributions of D3-branes which involved "ghosts" with negative tension and negative charge. If one formally uses these "ghostly" distributions to compute VEV's of the form $\left\langle\operatorname{tr} X_{\left(I_{1}\right.} \cdots X_{\left.I_{\ell}\right)}\right\rangle$, the results again violate inequalities among these VEV's that one can prove using the hermiticity of the fields $X_{I}$.

It would be interesting to describe more fully the subset $\mathcal{S} \subset$ $E_{6(6)} / U S p(8)$ on which the scalar potential is less than its value at the origin of the coset. $\mathcal{S}$ is far from being a uniform blob or half-space: it has tendrils which come arbitrarily close to subspaces of positive codimension as one proceeds further and further from the origin of the coset. We have proven that a static black hole horizon can only form at a location where the scalars lie in $\mathcal{S}$. The conjecture (2) essentially says that curvature singularities are allowed only if the scalars remain in $\mathcal{S}$ as one approaches the singularity. (Technically, we have not ruled out situations where $|\vec{\varphi}| \rightarrow \infty$ and $V(\vec{\varphi})$ approaches a constant value which is larger than $V$ at the origin of the coset. No example in the 
current literature has this feature, and it may be excludable on general grounds.)

A black hole horizon is the only purely geometrical expression of finite temperature. Black holes are also in the generic expression of finite temperature in a theory with gravity, in the sense that thermal excitations with enough energy should form a black hole. It sounds reasonable to claim that any finite temperature applied to a bulk geometry with a singularity where $g_{t t} \rightarrow 0$ will result in a black hole horizon cloaking the singularity, simply because the proper temperature diverges near the singularity. ${ }^{8}$ But it might happen that the horizon forms in a region very close to the singularity where curvatures are Planckian. Then low-energy supergravity will have limited value; indeed the very notion of horizon might have to be modified.

In an AdS/CFT context, it is straightforward to estimate when the five-dimensional supergravity treatment breaks down. In units where the radius of curvature of $A d S_{5}$ is unity, the five-dimensional Planck length scales as $N^{-2 / 3}$. In the case where the dual CFT is $\mathcal{N}=4$ superYang-Mills theory, $N$ is the rank of the gauge group; more generally, $N^{2}$ is the central charge of the dual CFT. Curvature invariants near a singularity of the form (29) become Planckian when $r-r_{0} \lesssim N^{-2 / 3}$. What is the entropy of a black hole horizon which forms just before curvatures become Planckian? The horizon area per unit volume at $r=r_{H}$ is $e^{3 A\left(r_{H}\right)}$. The entropy formula is actually more complicated than $S=A / 4 G_{5}$ when higher derivative terms become important $[66,67,68]$, but to obtain a rough-and-ready estimate we will stick with the simple area law. (For near-extremal D3-branes with no evolving scalars, the evidence of AdS/CFT is that all the $\alpha^{\prime}$ corrections only change the area law by $4 / 3$ in the $\alpha^{\prime} / L^{2} \rightarrow \infty$ limit $[69,70]$. However a "phase transition" at finite $\alpha^{\prime} / L^{2}$ cannot be ruled out [71].) In units where $L=1$, we have $1 / G_{5}=N^{2}$, so the entropy per unit volume is roughly $N^{2} e^{3 A\left(r_{H}\right)}$. Assuming that near-extremal solutions have nearly the same $A\left(r_{H}\right)$ as their Poincare invariant limit, one finds that the entropy per unit volume for a horizon at nearly Planckian curvatures scales as $N^{2-\frac{4}{3 \zeta^{2}}}$. This is to be compared with the expected scaling on the field theory side. If the field theory confines, then at low energy there are $O(1)$ degrees of freedom. A black hole horizon should have an $O(1)$ entropy if it purports to describe the theory at a temperature

\footnotetext{
${ }^{8}$ This line of argument was suggested to me by L. Susskind.
} 
much smaller than the confinement scale. This is possible if $\zeta<\sqrt{2 / 3}$, but precisely for this range there is no gap for excitations of a minimal scalar! We conclude that a five-dimensional supergravity treatment of near-extremal generalizations of singularities with $\zeta<\sqrt{2 / 3}$ should suffice to describe gapless field theory duals down to some minimum temperature which scales as an inverse power of $N$, but that the supergravity approximation is insufficient to describe the confining phase of a gauge theory. The original proposal of [61] avoids this problem by arriving at a confining gauge theory as the low-energy limit of a higher-dimensional gauge theory at finite temperature. However one is then left with a description where above the confinement scale the theory is higher dimensional.

We would like to think that the considerations of this paper will generalize to a string theoretic setting; that the conjecture (2) will carry over naturally to some more refined restriction on the nature of highly curved solutions; and that string theory considerations will provide a detailed "resolution" of the supergravity singularities which are allowed. In particular, D3-branes in an $H$-field should resolve the singularities found in section 4.3 (entries (F) and (G) in figure 3). With the supergravity solutions and the brane resolutions both in hand, one might make an interesting study of the transition between the deconfined phase (described by a black hole horizon in the supergravity solution) and the confined phase (described in terms of the brane resolution). It is conceivable that the transition will involve some dramatic alteration of the supergravity solution which we are simply not equipped to compute without a full understanding of the brane resolution. But we prefer the hopeful view that the low-energy theory, useful in so many ways as a guide to the qualitative features of the full string theory, will match more or less smoothly onto the microscopic description.

Matching a given brane resolution back onto supergravity seems sure to impose definite boundary conditions on the bulk fields near the singularity, but the process of finding these resolutions and extracting their supergravity limits could be tedious. Black hole solutions of the form (6) provide a quick and dirty route to establishing the boundary conditions at the singularity without going to the trouble of finding the brane resolution-provided one is interested in physics that is robust under finite temperature, and provided one is willing to grant that the low-energy supergravity description of finite temperature should match 
onto a microscopic description smoothly enough to distinguish good singularities from bad.

Here is the strategy. Suppose we have $n$ tachyonic scalars, corresponding to $n$ relevant operators that one might add to the microscopic lagrangian. Fix the more singular behavior of all these tachyons near the boundary of $A d S_{5}$ : this amounts to fixing the relevant deformations, but not the VEV's. Impose finite temperature in the form of a black hole horizon. The horizon boundary conditions (12) amount to $n$ more constraints on the solution. Assuming the form (9) for $A$ and $h$ (with $B$ left free, to be determined in terms of the temperature) amounts to using residual coordinate invariance to fix one boundary condition on $A$ and one on $h$ as $r \rightarrow \infty$. In total, if $T$ is regarded as fixed, there are $2 n+3$ constraints. There are $2 n+4$ integration constants of integration in the bulk equations of motion, (7), but the zero-energy constraint (8) fixes one. So the boundary value problem is exactly determined: generically there are solutions with a given temperature $T$, but only discretely many. ${ }^{9}$

As $T$ is lowered, there may be phase transitions, both first and second order, corresponding to discontinuities or bifurcations in the space of all finite temperature solutions. Sorting out such finite-temperature phase transitions would be a fascinating end in itself, which we have only delved into superficially with the prediction that $\alpha=1 / 2$ at a generic bifurcation. But let us suppose we have gotten past all the phase transitions, if necessary by switching our control parameter from $T$ to $B$ (see the footnote). As $B \rightarrow 0$, the horizon retreats into the singularity, but the scalars' evolution is still perfectly determined. In short, black holes of a given temperature impose just enough boundary conditions to be unique up to discrete choices, and singular Poincaré solutions which are limits of black holes inherit the same property.

In the case of a single scalar $\mu$, we can offer a definite conjecture regarding the nature of the condition at the singularity. Assume that $\mu$ becomes large and positive in solutions with small $T$ (or, more properly, small $B$ ), and that $V \sim-e^{\eta \mu}$ as $\mu \rightarrow \infty$ for some $\eta<\sqrt{32 / 3}$.

\footnotetext{
${ }^{9}$ At low temperatures it may become advantageous to use the non-extremality parameter $B$ as a control variable instead of temperature, since there are probably cases where $B$ is a multiple valued function of $T$ but not vice versa. $B$ scales as energy density above extremality.
} 
Then the Poincare limit of black hole solutions will have the form (29) with $\zeta=\eta / 2$. This is a very restrictive condition: the generic Poincaré invariant solution is of the form $(29)$ with $\zeta=\sqrt{8 / 3}$. Requiring the exceptional $\zeta=\eta / 2$ behavior amounts to imposing one boundary condition at the curvature singularity. We conjecture that Poincaré invariant solutions of this kind and only of this kind will have near-extremal generalizations. This is a much stronger statement than (2), and the evidence is slimmer. It could be phrased as a weak form of Cosmic Censorship: static nakedly singular solutions are allowed, but a theory with any thermalizable excitations will seek out the singularity with the smallest possible $\zeta$. The smallest possible $\zeta$ will always be less than $\sqrt{8 / 3}$ for potentials of the form (4).

Unfortunately, there is no solution involving only one scalar where we have a really clean understanding of the physics on the dual field theory side. However, we have run numerics to check the "Weak Cosmic Censorship" conjecture of the previous paragraph for two interesting cases. The first case is a deformation

$$
\mathcal{L} \rightarrow \mathcal{L}+\frac{1}{2} m^{2} \operatorname{tr}\left(-X_{1}^{2}-X_{2}^{2}-X_{3}^{2}-X_{4}^{2}+2 X_{5}^{2}+2 X_{6}^{2}\right)
$$

with $m^{2}>0$. The corresponding $V$ goes as $-e^{\sqrt{2 / 3} \mu}$ as $\mu \rightarrow \infty$ (see [8]). Black hole solutions appear to exist for any $T$, and as $T \rightarrow 0$ one does see a scaling region where the form (29) indeed appears with $\zeta=\sqrt{1 / 6}$ to $0.05 \%$ accuracy. Not unexpectedly, the VEV for $\mathcal{O}_{2}=\operatorname{tr}\left(-X_{1}^{2}-X_{2}^{2}-X_{3}^{2}-X_{4}^{2}+2 X_{5}^{2}+2 X_{6}^{2}\right)$ diverges as $T \rightarrow 0$. The second case is a deformation by a uniform mass for all three chiral adjoints - the case studied in section 4.3-but with only the scalar dual to the mass deformation excited, and not the scalar dual to the gaugino condensate. The scalar evolution is along the horizontal axis of figure 6(b). One might expect the Poincaré invariant solution to be only metastable, with a perturbative instability toward forming a gaugino condensate. In fact the normal modes of linear fluctuations of the supergravity scalar dual to the condensate do not include tachyons: the Schrodinger equation (58) in that case has the form of supersymmetric quantum mechanics with $\mathcal{Q}=\partial_{z}+3 \cot 2 z$, and the supersymmetric ground state corresponds to shifting to a neighboring trajectory in the plane of figure 6(b). Provisionally let us allow this case as a geometry which satisfies all the constraints we were able to put on solutions which should have field theory duals, even though the correct vacuum 
state on the field theory side remains obscure. At the least the solution is a candidate for verifying the conjectures of the previous paragraph, because $V(\mu) \sim-e^{\sqrt{16 / 3} \mu}$ and the Poincaré invariant, supersymmetric solution is of the form (29) with $\zeta=\sqrt{4 / 3}$. The numerics is somewhat stiffer in this case, but small $T$ solutions reveal scaling regions where $\zeta=\sqrt{4 / 3}$ can be verified to $3 \%$ accuracy.

In light of these numerical results, we feel entitled to a speculation regarding the marginal case $\zeta=\sqrt{8 / 3}$. This is in some sense the generic case, because if $V \sim-e^{\eta \mu}$ with $\eta<\sqrt{32 / 3}$, the generic Poincaré invariant solution is of the form (29) with $\zeta=\sqrt{8 / 3}$, whereas if $V \sim e^{\eta \mu}$ with $\eta>\sqrt{32 / 3}$, the condition (2) rules out all solutions. In the supersymmetric examples in this paper, we encountered $\zeta=\sqrt{8 / 3}$ twice: once in a Coulomb branch state of $\mathcal{N}=4$ gauge theory where the D3-branes were arranged in a perfect $S^{3}$ shell in the transverse dimensions; and again in a Coulomb branch state of a $\mathcal{N}=1$ mass deformation which had essentially the same $S^{3}$ shell interpretation. In both cases, the field theory dual makes it clear that the vacuum won't support finite temperature. If one does turn on finite temperature at the same time as introducing a term in the lagrangian of the form (62), then the trajectory may be nearly the same in the space of scalars, but the dependence on proper distance will be very different near the singularity: $\zeta=\sqrt{1 / 6}$ in the infrared, rather than $\sqrt{8 / 3}$. It is natural to speculate that the generic solutions with $\zeta=\sqrt{8 / 3}$ are allowed precisely when they correspond to exploring flat directions in the dual field theory, and that they can never support finite temperature. This unfortunately tells against some supergravity constructions which have been claimed to exhibit properties of confinement [72, 73, 74, 19], but the case for confinement in those examples was less than airtight.

It is particularly easy to show that the geometries of $[75,73]$, involving only the metric and the dilaton, cannot support a black hole horizon. The scalar equation of motion plus the horizon boundary conditions imply that the dilaton is everywhere constant. The only static black hole geometry involving only the metric and the dilaton is AdSSchwarzschild. This simple argument is another clue that singularities with $\zeta=\sqrt{8 / 3}$ cannot support finite $T$ : they have the same scaling as the solutions of $[75,73]$.

A feature of AdS/CFT which was essential to the proper interpre- 
tations of the examples in this paper is that a profile for a given scalar field can indicate either a deformation of the lagrangian or a VEV for some gauge singlet operator. This dual role of scalar profiles complicates any attempt to identify the value of a scalar at a given radius in $A d S_{5}$ with a coupling in an effective lagrangian for the dynamics at the corresponding scale. We must ask: 1) How do we disentangle the "VEV" part of the scalar profile from the "deformation" part? 2) Is such a disentanglement really necessary, or can the effective lagrangian be defined so as to pick out exactly the combination of VEV and deformation that AdS/CFT prescribes for its effective coupling? The issues might become clearer if one focused on solutions which are limits of black holes with prescribed behaviors for the scalar profiles corresponding to deformations of the microscopic lagrangian. The nearly unique specification of vacuum state in these solutions may simplify the interpretation of the holographic renormalization group proposed in [22]. For instance, the identification of beta functions with gradients of scalars [76, 22] seems odd in Coulomb branch states of $\mathcal{N}=4$ gauge theory, but possibly more natural for mass deformations in some preferred vacuum. A true acid test for the subject would be to reproduce some known quantitative features of the field theory RG, such as the NSVZ exact beta function.

\section{Acknowledgements}

This work would not have been possible without extensive communications with N. Warner, K. Pilch, and D. Freedman. I have also profited greatly from a number of exchanges with $\mathrm{R}$. Myers regarding near-extremal geometries, and with $\mathrm{H}$. Verlinde regarding the renormalization group in AdS/CFT. I also thank T. Banks, G. Horowitz, S. Kachru, I. Klebanov, B. Kol, V. Periwal, B. Pioline, J. Polchinski, S. Shenker, E. Silverstein, M. Strassler, L. Susskind, and E. Witten for useful discussions, and R. Myers for comments on an early draft. This research was supported in part by DOE grant DE-FG02-91ER40671 and by the Harvard Society of Fellows. 


\section{Appendix}

The purpose of this appendix is to remark on brane-world models in which there is a curvature singularity parallel to the brane on which visible sector matter exists, at some finite proper distance from it. Such constructions were first considered in the context of Horava-Witten theory in [77]. They have also appeared as a variant of the RandallSundrum construction (see for instance [78]). Following in the spirit of [79], it was recently proposed by two Stanford groups $[80,81]$ that such geometries could help solve the cosmological constant problem. In this appendix we will consider only the minimal case where the "Planck brane" is at one end of the spacetime, where $g_{t t}$ is finite, and the singularity is at the other, where $g_{t t} \rightarrow 0$. In the simplest constructions, where $g_{t t}$ is monotonic, gravity is in some sense localized on the Planck brane (hence the name). At least in most Horava-Witten theory constructions, the visible sector fields live on the Planck brane. The singularity then represents (or is resolved by) hidden sector fields which couple only gravitationally to the visible sector.

A crucial issue, both in the general scheme of [79] and in the specific proposal of $[80,81]$, is the boundary conditions at the singularity. At the level of classical five-dimensional gravity, the construction of $[80,81]$ is a boundary value problem, where one boundary is the Planck brane and the other boundary is a naked singularity. Similar analyses have appeared in many places, for example $[58,78,33]$. In [58, 33], spacetime ends on some orbifold fixed plane before $g_{t t} \rightarrow 0$, and boundary conditions are imposed both there and on the Planck brane. By contrast, in $[80,81]$, no boundary conditions are imposed at the singularity where $g_{t t} \rightarrow 0$. In $[58,33]$ (and other similar treatments) the boundary value problem is sufficiently determined to fix the four-dimensional cosmological constant, and there is no reason for it to be small. In [80, 81], there are fewer boundary conditions. The boundary value problem is underdetermined if the four-dimensional cosmological constant is left unspecified; it becomes determined (up to an multiplicative shift on the warp factor) if one specifies the four-dimensional cosmological constant. In particular, there do exist solutions with $3+1$-dimensional Poincaré invariance.

The issue which falls within the purview of this paper is whether the free boundary conditions used in $[80,81]$ are reasonable. Because 


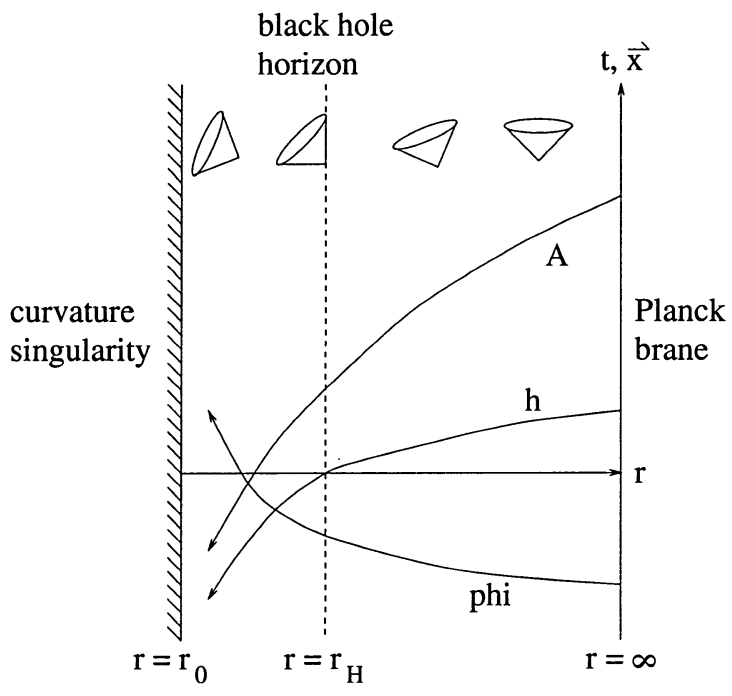

Figure 7: The spacetime described by (63). This is not a Penrose diagram: the lightcones tip over as indicated.

the solutions there purport to be at least a toy model for real world physics, they must be able to support finite temperature. We will interpret this as synonymous with being able to form a black horizon with finite Hawking temperature close to the singularity, without drastically changing the rest of the geometry. (Some potential pitfalls of this interpretation have been discussed in section 6). It is straightforward to show that a horizon is impossible in classical gravity if the bulk scalar is free, unless in fact the bulk scalar does not vary at all in the solution. Essentially the relevant observation has already been made in section 6 , but we will go into slightly more detail here to make the case clear. In order to have finite temperature, we seek a generalization of the Poincaré invariant solution of the form

$$
\begin{aligned}
d s^{2} & =e^{2 A(r)}\left(-h(r) d t^{2}+d \vec{x}^{2}\right)+d r^{2} / h(r) \\
\varphi & =\varphi(r)
\end{aligned}
$$

where $h(r)$ is a function which has a simple zero at $r=r_{H}$, the black hole horizon. The temperature is related to $h^{\prime}\left(r_{H}\right)$ (see (10)). A sketch of the geometry is presented in figure 7 .

The scalar equation of motion is (by assumption) $\square \varphi=0$ : explicitly,

$$
e^{-4 A} \partial_{r} e^{4 A} h \partial_{r} \varphi=0
$$


The geometry and the scalars should be perfectly regular at the horizon: an infalling observer must not notice anything special as he crosses through it. Thus in particular $\varphi^{\prime}$ and $e^{4 A}$ are finite at the horizon (as usual, primes denote derivatives with respect to $r$ ). So $e^{4 A} h \varphi^{\prime}=0$ at $r=r_{H}$. In view of (64), $e^{4 A} h \varphi^{\prime}=0$ everywhere. This is only possibility if $\varphi$ is in fact constant. Thus the original singular geometry of [80, 81], supported by a free scalar in the bulk, cannot be recovered as a limit of finite temperature black hole solutions. In [80], an intuitive picture of a "mechanism" by which the four-dimensional cosmological constant could be tuned to zero was presented in terms of a conserved current associated with the shift symmetry $\phi \rightarrow \phi+\delta \phi$ which. The current in some sense carries off visible sector vacuum energy into the bulk. This is an appealing picture, but in a geometry of the form (63) the current has the form $J^{r}=e^{4 A} h \partial_{r} \varphi$, which vanishes at the horizon and therefore throughout the geometry. ${ }^{10}$

We now wish to contemplate some variations on the basic Stanford proposal, still in the general framework of [79]. First, it is straightforward to work with any number of scalars with any bulk potential $V(\vec{\varphi})$ which can be written in the form

$$
V(\vec{\varphi})=\frac{1}{8}\left(\frac{\partial W}{\partial \vec{\varphi}}\right)^{2}-\frac{1}{3} W(\vec{\varphi})^{2} .
$$

Then a bulk geometry with $3+1$-dimensional Poincaré invariance can be generated from the first order equations (5), and it is possible that the naked singularities that generically arise can be obtained as limits of finite temperature black hole solutions provided $V$ is bounded above. Let us assume a Planck brane action of the form

$$
S_{\text {brane }}=-\int d^{4} \xi \sqrt{g_{(\text {induced })}} \lambda_{\mathrm{Pl}}(\vec{\varphi}) \text {. }
$$

The equations of motion that determine the possible embeddings of the Planck brane in the bulk geometry are

$$
\begin{aligned}
\theta_{i j}-\theta g_{i j}^{(\text {induced) }} & =\lambda_{\mathrm{Pl}}(\vec{\varphi}) g_{i j}^{\text {(induced) }} \\
\partial_{n} \vec{\varphi} & =\frac{1}{2} \frac{\partial \lambda_{\mathrm{Pl}}}{\partial \vec{\varphi}} .
\end{aligned}
$$

Here $\theta_{i j}$ is the extrinsic curvature of the Planck brane, $\theta$ is its trace, $g_{i j}^{\text {(induced) }}$ is the induced metric, and $\partial_{n}$ is the normal derivative at the

${ }^{10} \mathrm{I}$ thank M. Peskin and R. Sundrum for comments on this point. 
Planck brane. Equation (67) admits a solution where the induced metric is flat precisely if $\pm \lambda_{\mathrm{Pl}}(\vec{\varphi})$ is tangent to the $W(\vec{\varphi})$ used to generate the bulk geometry. Essentially this condition was obtained in [33].

The generalization of $[80]$ is to demand that $W(\vec{\varphi})= \pm \lambda_{\mathrm{Pl}}(\vec{\varphi})$ identically solves (65). When $V(\varphi)=0$ for a single scalar $\varphi$, this reduces to the special exponential $\lambda_{\mathrm{Pl}}(\varphi)$ considered explicitly in [80]. In HoravaWitten theory compactified on a Calabi-Yau manifold, with some embedding of the spin connection in one $E_{8}$, the tensions of the two ends of the universe do coincide, as functions of the Kahler moduli, with an appropriately defined bulk superpotential. This seeming coincidence is a result of supersymmetry. The corresponding no-force condition indeed guarantees the existence of solutions with $3+1$-dimensional Poincaré invariance. But it seems implausible that the tension of an end-of-theuniverse brane after supersymmetry breaking would solve the equation (65) identically.

The generalization of [81] is to demand only that $W(\vec{\varphi})$ and $\pm \lambda_{\mathrm{Pl}}(\vec{\varphi})$ are tangent at some point. This requires exactly one fine-tuning if $W(\vec{\varphi})$ is regarded as a fixed function of $\vec{\varphi}$. If there are $n$ scalars, then there is an $n$-parameter family of solutions to (65). If those $n$ parameters are left free, then there is an $(n-1)$-parameter family of solutions with $3+1$-dimensional Poincaré invariance both in the bulk and on the brane. Thus one "postpones" the cosmological constant problem from a single fine-tuning of the parameters of the theory to a single fine-tuning to the actual state of the universe (specified in this case by a choice of integration constants for (67)). It is not clear that there is any dynamical adjustment mechanism to push the universe toward a flat-space solution. (The current mentioned after (64) is only conserved in the case of a constant potential for some scalar).

On one hand, we should note that the conjecture (2), the fluctuation analysis in section 5, and the examples in sections 3.2 and 4 all point toward conditions at curvature singularities which take the form of inequalities rather than equalities. If this is the true state of affairs, and not just an indication of flat directions in the dual field theory or of an insufficiently precise understanding of the microscopic physics at the singularities, then the Stanford construction, as well as variations of it in the spirit of [79], are probably tenable. The only remaining proviso is that there is not in general a clear mechanism for preferring 
nearly flat solutions above all others.

On the other hand, if we believe that the bulk geometry should be the limit of a black hole solution (which seems particularly reasonable in a cosmological context, where the current state of the universe was arrived at through a long cooling process from very hot initial conditions), then the natural expectation is that boundary conditions at the naked singularity are inherited from its near-extremal generalizations. For instance, in the case of a free bulk scalar, the boundary condition in a static black hole geometry can be phrased as zero shift current across the horizon. Imposing this condition on naked singularities caused by a divergence in the bulk scalar simply rules out the existence of static singular solutions. At best these singularities could appear as transient states, probably with a lifetime comparable to the five-dimensional Planck time.

The construction of [78] is an excellent illustration of points we have argued. The singularity is of exactly the same type as the ones in $[80,81]$. In our language, the singularity is characterized by $\zeta=\sqrt{8 / 3}$. The only difference between [78] and $[80,81]$ is that in [78] there is a potential for the bulk scalar which goes to $-\infty$ at the singularity. The physics is transparent if we are willing to take the view that the bulk represents a cutoff quantum field theory, coupled to gravity by the existence of the Planck brane [82]. The bulk is precisely the fivedimensional representation of a state on the Coulomb branch of $\mathcal{N}=4$ gauge theory, corresponding to a ten-dimensional geometry where the D3-branes are arranged in an $S^{3}$ shell. This is a state which seems obviously incapable of supporting finite temperature. Because of the bulk potential, one might hope that the arguments following (64) have no force and that there are near-extremal generalizations. This is in fact one of the cases which we studied numerically (see discussion following (62)), with the conclusion that there are near-extremal geometries, but their extremal limit is a singularity with $\zeta=\sqrt{1 / 6}$. The non-generic value of $\zeta$ amounts to having precisely one boundary condition at the singularity, which is bad news because the problem of fitting a flat Planck brane to the bulk geometry is once again fine-tuned by one real parameter!

According to what was referred to the "Weak Cosmic Censorship" conjecture in section 6 , the situation of the previous paragraph is 
generic. If weak cosmic censorship is right, then singularities of the form (29) with $\zeta \geq \sqrt{8 / 3}$ cannot be obtained as limits of regular black holes. This seems like only a slightly stronger statement than what we proved in sections 3.1 and 3.3 , but in fact $\zeta=\sqrt{8 / 3}$ is both a hard case to settle and a common one in examples. Our evidence for ruling it out is 1) numerics on two examples, 2) the calculations following (64), and 3) the field theory intuition of the previous paragraph. This is not an airtight case. However, the conjecture that singularities with $\zeta \geq \sqrt{8 / 3}$ do not admit near-extremal generalizations is mathematically well-posed and capable of proof or disproof.

From the point of view of a boundary value problem, the boundary conditions at the Planck brane, (67), have essentially the same character as the boundary conditions at the true boundary of $A d S_{5}$ that correspond to fixing the microscopic lagrangian. Replacing the naked singularity either with a negative tension brane or with a black hole horizon imposes enough boundary conditions to completely determine the boundary value problem. The cosmological constant is not free, but fixed, and it still seems accidental that its value in four-dimensional Planck units is as small as $10^{-120}$. Going on the AdS/CFT intuition that the naked singularity represents interesting infrared dynamics of a quantum field theory, it seems obvious that supersymmetry breaking should induce a positive cosmological constant. It would be fascinating if this intuition were somehow wrong, and if the naked singularity could be somehow associated with a breaking of supersymmetry that does not lead to a four-dimensional cosmological constant. To realize this hope in a convincing and coherent model would require some new ideas.

Constructions such as those in [80, 81], and near-extremal generalizations of them are nevertheless interesting. If the Planck brane's properties can somehow be fine-tuned so that a flat solution can be obtained, then black hole generalizations of the Poincaré invariant solution allow one to obtain interesting spatially flat FRW metrics. The mass density above extremality of the black hole will make a contribution to $(\dot{a} / a)^{2}$ in the Friedmann equation. This was demonstrated in detail in [82] for the case of an $A d S_{5}$ bulk, and has been considered in more generality in $[83,84,85,86,87]$. The black hole geometry represents hidden sector degrees of freedom at finite temperature. 


\section{References}

[1] J. Maldacena, The Large $N$ limit of superconformal field theories and supergravity, Adv. Theor. Math. Phys., 2 (1998), 231, hep-th/9711200.

[2] S.S. Gubser, I.R. Klebanov, and A.M. Polyakov, Gauge theory correlators from noncritical string theory, Phys. Lett., B428 (1998), 105, hep-th/9802109.

[3] E. Witten, Anti-de Sitter space and holography, Adv. Theor. Math. Phys., 2 (1998), 253, hep-th/9802150.

[4] O. Aharony, S.S. Gubser, J. Maldacena, H. Ooguri, and Y. Oz, Large $N$ field theories, string theory and gravity, hep-th/9905111.

[5] G.T. Horowitz and R. Myers, The value of singularities, Gen. Rel. Grav., 27 (1995), 915, gr-qc/9503062.

[6] P. Kraus, F. Larsen, and S.P. Trivedi, The Coulomb branch of gauge theory from rotating branes, JHEP, 03 (1999), 003, hep-th/9811120.

[7] I.R. Klebanov and E. Witten, AdS/CFT correspondence and symmetry breaking, Nucl. Phys., B556 (1999), 89, hep-th/9905104.

[8] D.Z. Freedman, S.S. Gubser, K. Pilch, and N.P. Warner, Continuous distributions of D3-branes and gauged supergravity, hep-th/9906194.

[9] A. Brandhuber and K. Sfetsos, Wilson loops from multicentre and rotating branes, mass gaps and phase structure in gauge theories, hep-th/9906201.

[10] M. Cvetic, S.S. Gubser, H. Lu, and C.N. Pope, Symmetric potentials of gauged supergravities in diverse dimensions and Coulomb branch of gauge theories, hep-th/9909121.

[11] I.R. Klebanov and E. Witten, Superconformal field theory on threebranes at a Calabi-Yau singularity, Nucl. Phys., B536 (1998), 199, hep-th/9807080. 
[12] S. Gubser, N. Nekrasov, and S. Shatashvili, Generalized conifolds and four dimensional $N=1$ superconformal theories, JHEP, 05 (1999), 003, hep-th/9811230.

[13] E. Lopez, A family of $N=1 S U(N)^{* *} k$ theories from branes at singularities, JHEP, 02 (1999), 019, hep-th/9812025.

[14] L. Girardello, M. Petrini, M. Porrati, and A. Zaffaroni, Novel local CFT and exact results on perturbations of $N=4$ super Yang-Mills from AdS dynamics, JHEP, 12 (1998), 022, hep-th/9810126.

[15] J. Distler and F. Zamora, Non-supersymmetric conformal field theories from stable anti-de Sitter spaces, Adv. Theor. Math. Phys., 2 (1999), 1405, hep-th/9810206.

[16] A. Khavaev, K. Pilch, and N.P. Warner, New vacua of gauged $N$ $=8$ supergravity in five dimensions, hep-th/9812035.

[17] A. Karch, D. Lust, and A. Miemiec, New $N=1$ superconformal field theories and their supergravity description, Phys. Lett., B454 (1999), 265, hep-th/9901041.

[18] D.Z. Freedman, S.S. Gubser, K. Pilch, and N.P. Warner, Renormalization group flows from holography supersymmetry and a ctheorem, hep-th/9904017.

[19] L. Girardello, M. Petrini, M. Porrati, and A. Zaffaroni, Confinement and condensates without fine tuning in supergravity duals of gauge theories, JHEP, 05 (1999), 026, hep-th/9903026.

[20] L. Girardello, M. Petrini, M. Porrati, and A. Zaffaroni, The supergravity dual of $N=1$ superYang-Mills theory, hep-th/9909047.

[21] S.S. Gubser, Non-conformal examples of AdS/CFT, hep-th/9910117.

[22] J. de Boer, E. Verlinde, and H. Verlinde, On the holographic renormalization group, hep-th/9912012.

[23] I.R. Klebanov and N.A. Nekrasov, Gravity duals of fractional branes and logarithmic $R G$ flow, hep-th/9911096. 
[24] I. Chepelev and R. Roiban, A note on correlation functions in AdS(5)/SYM(4) correspondence on the Coulomb branch, Phys. Lett., B462 (1999), 74-80, hep-th/9906224.

[25] S.B. Giddings and S.F. Ross, D3-brane shells to black branes on the Coulomb branch, Phys. Rev., D61 (2000), 024036, hep-th/9907204.

[26] A. Lukas, B.A. Ovrut, and D. Waldram, Heterotic M-theory vacua with five-branes, hep-th/9903144.

[27] L. Susskind and E. Witten, The holographic bound in anti-de Sitter space, hep-th/9805114.

[28] A.W. Peet and J. Polchinski, UV/IR relations in AdS dynamics, Phys. Rev., D59 (1999), 065011, hep-th/9809022.

[29] M. Gunaydin, L.J. Romans, and N.P. Warner, Compact and noncompact gauged supergravity theories in five-dimensions, Nucl. Phys., B272 (1986), 598.

[30] M. Pernici, K. Pilch, and P. van Nieuwenhuizen, Gauged N=8 D=5 supergravity, Nucl. Phys., B259 (1985), 460.

[31] R.C. Myers, unpublished notes, April 1999.

[32] K. Skenderis and P.K. Townsend, Gravitational stability and renormalization-group flow, Phys. Lett., B468 (1999), 46, hep-th/9909070.

[33] O. DeWolfe, D.Z. Freedman, S.S. Gubser, and A. Karch, Modeling the fifth dimension with scalars and gravity, hep-th/9909134.

[34] P. Breitenlohner and D.Z. Freedman, Stability in gauged extended supergravity, Ann. Phys., 144 (1982), 249.

[35] L. Mezincescu and P.K. Townsend, Atability at a local maximum in higher dimensional anti-de Sitter space and applications to supergravity, Ann. Phys., 160 (1985), 406.

[36] I. Bakas and K. Sfetsos, States and curves of five-dimensional gauged supergravity, hep-th/9909041. 
[37] C.V. Johnson, A.W. Peet, and J. Polchinski, Gauge theory and the excision of repulson singularities, hep-th/9911161.

[38] G.T. Horowitz and J. Polchinski, A correspondence principle for black holes and strings, Phys. Rev., D55 (1997), 6189-6197, hep-th/9612146.

[39] T. Appelquist, A.G. Cohen, and M. Schmaltz, A new constraint on strongly coupled field theories, Phys. Rev., D60 (1999), 045003, hep-th/9901109.

[40] S.S. Gubser, K. Pilch, and N. Warner, unpublished.

[41] M. Cvetic and S.S. Gubser, Phases of R-charged black holes, spinning branes and strongly coupled gauge theories, JHEP, 04 (1999), 024, hep-th/9902195.

[42] A. Chamblin, R. Emparan, C.V. Johnson, and R.C. Myers, Charged AdS black holes and catastrophic holography, Phys. Rev., D60 (1999), 064018, hep-th/9902170.

[43] S.S. Gubser, Thermodynamics of spinning D3-branes, Nucl. Phys., B551 (1999), 667, hep-th/9810225.

[44] R. Donagi and E. Witten, Supersymmetric Yang-Mills theory and integrable systems, Nucl. Phys., B460 (1996), 299-334, hep-th/9510101.

[45] N. Seiberg and E. Witten, Monopoles, duality and chiral symmetry breaking in $N=2$ supersymmetric QCD, Nucl. Phys., B431 (1994), 484-550, hep-th/9408099.

[46] P.C. Argyres and A.E. Faraggi, The vacuum structure and spectrum of $N=2$ supersymmetric $S U(n)$ gauge theory, Phys. Rev. Lett., 74 (1995), 3931-3934, hep-th/9411057.

[47] A. Klemm, W. Lerche, S. Yankielowicz, and S. Theisen, Simple singularities and $N=2$ supersymmetric Yang-Mills theory, Phys. Lett., B344 (1995), 169-175, hep-th/9411048.

[48] C. Vafa and E. Witten, A Strong coupling test of $S$ duality, Nucl. Phys., B431 (1994), 3-77, hep-th/9408074.

[49] R.C. Myers, Dielectric-branes, hep-th/9910053. 
[50] V.A. Novikov, M.A. Shifman, A.I. Vainshtein, and V.I. Zakharov, Instanton effects in supersymmetric theories, Nucl. Phys., B229 (1983), 407.

[51] M.A. Shifman and A.I. Vainshtein, On gluino condensation in supersymmetric gauge theories. $S U(N)$ and $O(N)$ groups, Nucl. Phys., B296 (1988), 445.

[52] K. Konishi, Anomalous supersymmetry transformation of some composite operators in SQCD, Phys. Lett., B135 (1984), 439.

[53] K. Intriligator, R.G. Leigh, and N. Seiberg, Exact superpotentials in four-dimensions, Phys. Rev., D50 (1994), 1092-1104, hep-th/9403198.

[54] K. Intriligator and N. Seiberg, Lectures on supersymmetric gauge theories and electric- magnetic duality, Nucl. Phys. Proc. Suppl., 45BC (1996), 1-28, hep-th/9509066.

[55] M. Shifman, Exact results in gauge theories: Putting supersymmetry to work, hep-th/9906049.

[56] E. Witten, Branes and the dynamics of QCD, Nucl. Phys., B507 (1997), 658, hep-th/9706109.

[57] N. Dorey and S.P. Kumar, Softly-broken $N=4$ supersymmetry in the large- $N$ limit, hep-th/0001103.

[58] A. Lukas, B.A. Ovrut, K.S. Stelle, and D. Waldram, The universe as a domain wall, Phys. Rev., D59 (1999), 086001, hep-th/9803235.

[59] K. Pilch, unpublished.

[60] J. Distler and F. Zamora, Chiral symmetry breaking in the AdS/CFT correspondence, hep-th/9911040.

[61] E. Witten, Anti-de Sitter space, thermal phase transition, and confinement in gauge theories, Adv. Theor. Math. Phys., 2 (1998), 505 , hep-th/9803131.

[62] C. Csaki, H. Ooguri, Y. Oz, and J. Terning, Glueball mass spectrum from supergravity, JHEP, 01 (1999), 017, hep-th/9806021. 
[63] S.S. Gubser, Non-conformal examples of AdS/CFT, hep-th/9910117.

[64] R.M. Wald, Dynamics in nonglobally hyperbolic, static space-times, J. Math. Phys., 21 (1980), 2802.

[65] G.T. Horowitz and D. Marolf, Quantum probes of space-time singularities, Phys. Rev., D52 (1995), 5670-5675, gr-qc/9504028.

[66] T. Jacobson and R.C. Myers, Black hole entropy and higher curvature interactions, Phys. Rev. Lett., 70 (1993), 3684-3687, hep-th/9305016.

[67] T. Jacobson, G. Kang, and R.C. Myers, On black hole entropy, Phys. Rev., D49 (1994), 6587-6598, gr-qc/9312023.

[68] V. Iyer and R.M. Wald, Some properties of Noether charge and a proposal for dynamical black hole entropy, Phys. Rev., D50 (1994), 846-864, gr-qc/9403028.

[69] S.S. Gubser, I.R. Klebanov, and A.W. Peet, Entropy and Temperature of Black 3-Branes, Phys. Rev., D54 (1996), 3915-3919, hep-th/9602135.

[70] S.S. Gubser, I.R. Klebanov, and A.A. Tseytlin, Coupling constant dependence in the thermodynamics of $N=4$ supersymmetric YangMills theory, Nucl. Phys., B534 (1998), 202, hep-th/9805156.

[71] M. Li, Evidence for large $N$ phase transition in $N=4$ super Yang- Mills theory at finite temperature, JHEP, 03 (1999), 004, hep-th/9807196.

[72] J.A. Minahan, Asymptotic freedom and confinement from type 0 string theory, JHEP, 04 (1999), 007, hep-th/9902074.

[73] S.S. Gubser, Dilaton-driven confinement, hep-th/9902155.

[74] A. Kehagias and K. Sfetsos, On asymptotic freedom and confinement from type-IIB supergravity, Phys. Lett., B456 (1999), 22, hep-th/9903109.

[75] A. Kehagias and K. Sfetsos, On running couplings in gauge theories from type-IIB supergravity, Phys. Lett., B454 (1999), 270, hep-th/9902125. 
[76] K. Behrndt, Domain walls of $D=5$ supergravity and fixpoints of $N=1$ super Yang-Mills, hep-th/9907070.

[77] E. Witten, Strong Coupling Expansion Of Calabi-Yau Compactification, Nucl. Phys., B471 (1996), 135-158, hep-th/9602070.

[78] A. Brandhuber and K. Sfetsos, Non-standard compactifications with mass gaps and Newton's law, JHEP, 10 (1999), 013, hep-th/9908116.

[79] E. Verlinde and H. Verlinde, $R G$-flow, gravity and the cosmological constant, hep-th/9912018.

[80] N. Arkani-Hamed, S. Dimopoulos, N. Kaloper, and R. Sundrum, A small cosmological constant from a large extra dimension, hep-th/0001197.

[81] S. Kachru, M. Schulz, and E. Silverstein, Self-tuning flat domain walls in 5d gravity and string theory, hep-th/0001206.

[82] S.S. Gubser, AdS/CFT and gravity, hep-th/9912001.

[83] A. Kehagias and E. Kiritsis, "Mirage cosmology," JHEP 11 (1999) 022, hep-th/9910174.

[84] P. Kraus, "Dynamics of anti-de Sitter domain walls," hep-th/9910149.

[85] T. Shiromizu, K. Maeda, and M. Sasaki, The Einstein equation on the 3-brane world, gr-qc/9910076.

[86] P. Binetruy, C. Deffayet, U. Ellwanger, and D. Langlois, Brane cosmological evolution in a bulk with cosmological constant, hep-th/9910219.

[87] S. Mukohyama, Brane-world solutions, standard cosmology, and dark radiation, hep-th/9911165. 